\title{
Deformation quantisation for $(-1)$-shifted symplectic structures and vanishing cycles
}

\author{
J. P. Pridham
}

\begin{abstract}
We formulate a notion of $E_{0}$ quantisation of (-1)-Poisson structures on derived Artin $N$-stacks and construct a map from $E_{0}$ quantisations of $(-1)$-shifted symplectic structures to power series in de Rham cohomology. For a square root of the dualising line bundle, this gives an equivalence between even power series and self-dual quantisations. In particular, there is a canonical quantisation of any such square root, which localises to recover the perverse sheaf of vanishing cycles on derived Deligne-Mumford stacks, thus giving a form of derived categorification of Donaldson-Thomas invariants.
\end{abstract}

\section{Introduction}

In [PTVV13], the notion of shifted symplectic structures on derived stacks was introduced. Examples of derived stacks carrying ( -1$)$-shifted symplectic structures are Lagrangian intersections and character varieties of threefolds. In $\left[\mathrm{BBD}^{+} 15\right]$, a perverse sheaf of vanishing cycles was then constructed on any oriented $(-1)$-shifted symplectic derived $\mathbb{C}$-scheme, giving a categorification of Behrend's function [Beh09], and hence of Donaldson-Thomas invariants when applied to the derived moduli stack of sheaves on a Calabi-Yau threefold.

As discussed in [Toë14], there is a general philosophy that $n$-shifted symplectic structures should give rise to $E_{n+1}$-algebra quantisations of the structure sheaf. For $n=-1$, this means some sort of deformation as an abelian group, and there was an expectation that this should be related to the perverse sheaf $\mathcal{P} \mathcal{V}$ of $\left[\mathrm{BBD}^{+} 15\right]$ and to Batalin-Vilkovisky (BV for short) algebras as in [Beh14, $\S 3.4 .3]$. In this paper, we realise these expectations by formulating and establishing $E_{0}$ quantisation as a genuinely derived object for each oriented (-1)-shifted symplectic structure and show that its localisation recovers $\mathcal{P} \mathcal{V}$.

A (-1)-shifted Poisson structure on a derived scheme $X$ is a Maurer-Cartan element $\pi=$ $\sum_{i \geqslant 2} \pi_{i}$ with $\pi_{i} \in \operatorname{Symm}^{i} \mathscr{T}_{X}$. For a line bundle $\mathscr{L}$, we define an $E_{0}$ quantisation of $(\pi, \mathscr{L})$ to be a Maurer-Cartan element $\Delta=\sum_{i \geqslant 2} \Delta_{i} \hbar^{i-1} \in \mathscr{D}_{X}(\mathscr{L}) \llbracket \hbar \rrbracket$ such that $\Delta_{i}$ is a differential operator of order $i$ lifting $\pi_{i}$.

The proof in [Pri17] of the correspondence between $n$-shifted symplectic and non-degenerate Poisson structures relied on the existence, for all Poisson structures $\pi$, of a map $\mu(-, \pi)$ from

Received 22 May 2017, accepted in final form 31 December 2018.

2010 Mathematics Subject Classification 14F05 (primary), 14A20, 18G30, 32S30 (secondary).

Keywords: deformation quantisation, shifted symplectic structures, vanishing cycles.

This journal is (C) Foundation Compositio Mathematica 2019. This article is distributed with Open Access under the terms of the Creative Commons Attribution Non-Commercial License, which permits non-commercial reuse, distribution, and reproduction in any medium, provided that the original work is properly cited. For commercial re-use, please contact the Foundation Compositio Mathematica.

This work was supported by the Engineering and Physical Sciences Research Council [grant number $\mathrm{EP} / \mathrm{I} 004130 / 2]$. 


\section{J. P. PRIDHAM}

the de Rham algebra to the algebra $T_{\pi} \widehat{\operatorname{Pol}}(X, n)$ of shifted polyvectors with differential twisted by $\pi$. Since $[\pi,-]$ defines a derivation from $\mathscr{O}_{X}$ to $T_{\pi} \widehat{\operatorname{Pol}}(X, n)$, it determines a map $\Omega_{X}^{1} \rightarrow$ $T_{\pi} \widehat{\operatorname{Pol}}(X, n)[1]$, and $\mu(-, \pi)$ is the resulting morphism of graded-commutative differential algebras (CDGAs for short).

We adapt this idea to construct (Lemma 1.32), for any $E_{0}$ quantisation $\Delta$ of a CDGA $A$, an $A_{\infty}$-morphism $\mu(-, \Delta)$ from the de Rham algebra $\operatorname{DR}(A)$ to $\mathscr{D}_{A} \llbracket \hbar \rrbracket$. Roughly speaking, this is an $A$-algebra homomorphism with the restriction to $\Omega_{A}^{1}$ corresponding to the derivation $[\Delta,-]$; because $\mathscr{D}_{A}$ is not commutative, we have to define $\mu$ explicitly on an associative algebra resolution of the de Rham algebra.

This gives rise to a notion of compatibility between $E_{0}$ quantisations $\Delta$ and generalised (-1)shifted pre-symplectic structures (power series $\omega$ of elements of the de Rham complex): we say that $\omega$ and $\Delta$ are compatible if

$$
\mu(\omega, \Delta) \simeq \hbar^{2} \frac{\partial \Delta}{\partial \hbar} .
$$

Proposition 1.38 shows that every non-degenerate affine quantisation $\Delta$ has a unique compatible generalised pre-symplectic structure, thus giving us a map

$$
Q \mathcal{P}(A,-1)^{\text {nondeg }} \rightarrow \mathrm{H}^{1}\left(F^{2} \mathrm{DR}(A)\right) \times \hbar \mathrm{H}^{1}(\mathrm{DR}(A)) \llbracket \hbar \rrbracket
$$

on the space of non-degenerate $E_{0}$ quantisations.

In fact, much more is true. We have spaces $Q \mathcal{P}(A,-1) / G^{k+1}$ consisting of $E_{0}$ quantisations of order $k$, by which we mean Maurer-Cartan elements in $\prod_{j \geqslant 2}\left(F_{j} \mathscr{D}_{A} / F_{j-k-1}\right) \hbar^{j-1}$, for $F$ the order filtration on $\mathscr{D}$. We then have maps

$$
Q \mathcal{P}(A,-1)^{\text {nondeg }} / G^{k+1} \rightarrow \mathrm{H}^{1}\left(F^{2} \mathrm{DR}(A)\right) \times \hbar \mathrm{H}^{1}(\mathrm{DR}(A))[\hbar] / \hbar^{k},
$$

and Proposition 1.41 shows that the resulting map

$$
Q \mathcal{P}(A,-1)^{\text {nondeg }} \rightarrow\left(Q \mathcal{P}(A,-1)^{\text {nondeg }} / G^{2}\right) \times \hbar^{2} \mathrm{H}^{1}(\mathrm{DR}(A)) \llbracket \hbar \rrbracket
$$

underlies an equivalence. Thus quantisation reduces to a first-order problem.

Section 2 interposes some abstract nonsense to transfer these results from affine derived schemes to derived Deligne-Mumford (DM) N-stacks (Propositions 2.23 and 2.20). Section 3 extends the results of Section 1 to the formalism of commutative bidifferential bigraded algebras, and thus to derived Artin $N$-stacks (Proposition 3.14).

When $\mathscr{L}$ is Grothendieck-Verdier self-dual (that is, a square root of the dualising line bundle $\left.\omega_{X}\right)$ or, more generally, whenever $\mathscr{D}(\mathscr{L}) \simeq \mathscr{D}(\mathscr{L})^{\text {opp}}$, Section 4 introduces a notion of self-duality for quantisations $\Delta$ of $\mathscr{L}$. For self-dual quantisations, the first-order obstruction vanishes, and in fact the equivalence class of such quantisations of a non-degenerate (-1)-shifted Poisson structure is canonically isomorphic to

$$
\hbar^{2} \mathrm{H}_{\mathrm{dR}}^{1}(X) \llbracket \hbar^{2} \rrbracket .
$$

In particular, there is an $\infty$-functor from the space of $(-1)$-shifted symplectic structures to deformation quantisations of $\mathscr{L}$; for the symplectic structure on a derived critical locus, this quantisation is just given by a twisted Hodge complex, so (Proposition 4.9) localising at $\hbar$ recovers the perverse sheaf of vanishing cycles studied in $\left[\mathrm{BBD}^{+} 15\right]$.

In Section 4.3, we discuss how to adapt these results to quantisation of $n$-shifted symplectic structures for $n \geqslant-2$. For $n=-2$, quantisations should arise as Maurer-Cartan elements of a $B V$-algebra quantisation of $(-2)$-shifted polyvectors. For $n \geqslant 0$, the formality of the $E_{n+2}$ operad allows the construction of a compatibility map $\mu$ from de Rham cohomology to quantised 


\section{QUANTISING (-1)-SHIFTED SYMPLECTIC STRUCTURES}

Poisson cohomology, leading to a map from non-degenerate $E_{n+1}$ quantisations of $\mathscr{O}_{X}$ to power series

$$
\mathrm{H}^{n+2}\left(F^{2} \mathrm{DR}(A)\right) \times \hbar \mathrm{H}^{n+2}(\mathrm{DR}(A)) \llbracket \hbar \rrbracket .
$$

Again, the only obstruction to deforming a non-degenerate Poisson structure is of first order, and we sketch a notion of self-duality under which the obstruction vanishes - for details, see [Pri18a].

\section{Compatible quantisations on derived affine schemes}

In this section, we develop the notion of compatibility between $E_{0}$ quantisations and generalised $(-1)$-shifted pre-symplectic structures on derived affine schemes, ultimately reducing deformation quantisation to a first-order problem.

Let $R$ be a CDGA over $\mathbb{Q}$, and fix a CDGA $A$ over $R$. We will denote the differentials on $A$ and $R$ by $\delta$. Given $R$-modules $M$ and $N$ in cochain complexes, we write $\underline{\operatorname{Hom}}_{R}(M, N)$ for the cochain complex given by

$$
\underline{\operatorname{Hom}}_{R}(M, N)^{i}=\operatorname{Hom}_{R \#}\left(M^{\#}, N^{\#[i]}\right)
$$

with differential $\delta f=\delta_{N} \circ f \pm f \circ \delta_{M}$, where $V^{\#}$ denotes the graded module underlying a cochain complex $V$.

1.1. Differential operators and quantised polyvectors. We now formulate a theory of differential operators for our CDGA $A$, leading to a notion of deformation quantisation for a $(-1)$-shifted Poisson structure.

\subsubsection{Differential operators}

Definition 1.1. Given $A$-modules $M$ and $N$ in cochain complexes, inductively define the filtered cochain complex $\operatorname{Diff}(M, N)=\operatorname{Diff}_{A / R}(M, N) \subset \underline{\operatorname{Hom}}_{R}(M, N)$ of differential operators from $M$ to $N$ by setting

(1) $F_{0} \operatorname{Diff}(M, N)=\underline{\operatorname{Hom}}_{A}(M, N)$;

(2) $F_{k+1} \operatorname{Diff}(M, N)=\left\{u \in \underline{\operatorname{Hom}}_{R}(M, N):[a, u] \in F_{k} \operatorname{Diff}(M, N) \forall a \in A\right\}$, where $[a, u]=$ $a u-(-1)^{\operatorname{deg} a \operatorname{deg} u} u a$;

(3) $\operatorname{Diff}(M, N)=\lim _{k} F_{k} \operatorname{Diff}(M, N)$.

(The reason for the notation $F$ is that $F^{p}:=F_{-p}$ gives a Hodge filtration.)

The definitions ensure that the associated graded pieces $\operatorname{gr}_{k}^{F} \operatorname{Diff}_{A}(M, N)$ have the structure of $A$-modules. Also note that for any $u \in F_{k+1} \operatorname{Diff}(M, N)$, the commutator $[u,-]$ defines a derivation from $A$ to $\operatorname{gr}_{k}^{F} \operatorname{Diff}(M, N)$, giving an $A$-linear map

$$
\operatorname{gr}_{k+1}^{F} \operatorname{Diff}(M, N) \rightarrow \underline{\operatorname{Hom}}_{A}\left(\Omega_{A}^{1}, \operatorname{gr}_{k}^{F} \operatorname{Diff}(M, N)\right) .
$$

Proceeding inductively and considering the symmetries involved, this gives maps

$$
\operatorname{gr}_{k}^{F} \operatorname{Diff}(M, N) \rightarrow \underline{\operatorname{Hom}}_{A}\left(M \otimes_{A} \operatorname{CoS}_{A}^{k} \Omega_{A}^{1}, N\right)
$$

for all $k$. $\left(\right.$ Here, $\operatorname{CoS}_{A}^{p}(M)=\operatorname{CoSymm}_{A}^{p}(M)=\left(M^{\otimes_{A} p}\right)^{\Sigma_{p}}$ and $\operatorname{CoSymm}_{A}(M)=\bigoplus_{p \geqslant 0} \operatorname{CoS}_{A}^{p}(M)$.)

These maps will be isomorphisms whenever $A$ is semi-smooth in the sense that the underlying graded algebra $A^{\#}$ is isomorphic to $\left(R^{\#} \otimes_{R^{0}} S\right)\left[P^{\#}\right]$, for $S$ a smooth $R^{0}$-algebra and $P^{\#}$ a graded 


\section{J. P. PRIDHAM}

projective module over $R^{\#} \otimes_{R^{0}} S$ (in particular, if $A$ is cofibrant as a CDGA over $R$ ), and $M^{\#}$ is projective over $A^{\#}$.

Also observe that for $A$-modules $M, N, P$, the composition $\operatorname{map} \underline{\operatorname{Hom}}_{R}(N, P) \otimes_{R} \underline{\operatorname{Hom}}_{R}(M, N)$ $\stackrel{\circ}{\rightarrow} \underline{\operatorname{Hom}}_{R}(M, P)$ restricts to give $F_{l} \operatorname{Diff}_{A / R}(N, P) \otimes_{R} F_{k} \operatorname{Diff}_{A / R}(M, N) \rightarrow F_{k+l} \operatorname{Diff}_{A / R}(M, P)$.

Definition 1.2. Given an $A$-module $M$ in cochain complexes, write $\mathscr{D}(M)=\mathscr{D}_{A / R}(M):=$ $\operatorname{Diff}_{A / R}(M, M)$, which we regard as a differential graded augmented algebra (DGAA for short) under the composition above. We simply write $\mathscr{D}_{A}=\mathscr{D}_{A / R}$ for $\mathscr{D}_{A / R}(A, A)$.

For $A$-modules $M$ and $N$, inclusion in $\operatorname{Hom}_{R}(M, N)$ gives a natural map $\underline{\operatorname{Hom}}_{A}\left(M,\left(N \otimes_{A}\right.\right.$ $\left.\left.\mathscr{D}_{A}\right)^{r}\right) \rightarrow \operatorname{Diff}(M, N)$, where $(-)^{r}$ denotes the right $A$-module structure. This will be an isomorphism whenever $A$ is semi-smooth over $R$ (in particular, if $A$ is cofibrant as a CDGA over $R)$.

Remark 1.3. For $A$ semi-smooth over $R$, there is an equivalent alternative description of $\operatorname{Diff}(M, N)$, familiar from the underived setting. The algebra $A \otimes_{R} A$ is naturally an $A$-bimodule, and if we write $I$ for the diagonal ideal $\operatorname{ker}\left(A \otimes_{R} A \rightarrow A\right)$, then there are natural isomorphisms

$$
\alpha: \underline{\operatorname{Hom}}_{A}(\left(A \otimes_{R} M\right) / \overbrace{I \cdots I}^{k+1} M, N) \rightarrow F_{k} \operatorname{Diff}(M, N),
$$

given by $\alpha(f)(m):=f(1 \otimes m)$.

1.1.2. Polyvectors. The following is adapted from [Pri17, Definition 1.1], with the introduction of a dummy variable $\hbar$ of cohomological degree 0 .

Definition 1.4. Define the complex of $(-1)$-shifted polyvector fields on $A$ by

$$
\widehat{\operatorname{Pol}}(A / R,-1):=\prod_{p \geqslant 0} \underline{\operatorname{Hom}}_{A}\left(\operatorname{CoS}_{A}^{p}\left(\Omega_{A / R}^{1}\right), A\right) \hbar^{p-1}
$$

with graded-commutative multiplication $(a, b) \mapsto \hbar a b$ following the usual conventions for symmetric powers.

The Lie bracket on $\operatorname{Hom}_{A}\left(\Omega_{A / R}^{1}, A\right)$ then extends to give a bracket (the Schouten-Nijenhuis bracket)

$$
[-,-]: \widehat{\operatorname{Pol}}(A / R,-1) \times \widehat{\operatorname{Pol}}(A / R,-1) \rightarrow \widehat{\operatorname{Pol}}(A / R,-1),
$$

determined by the property that it is a bi-derivation with respect to the multiplication operation.

Thus $\widehat{\operatorname{Pol}}(A / R,-1)$ has the natural structure of a $P_{1}$-algebra (that is, a Poisson algebra), and in particular $\widehat{\operatorname{Pol}}(A / R,-1)$ is a differential graded Lie algebra (DGLA for short) over $R$. Note that the differential $\delta$ on $\widehat{\operatorname{Pol}}(A / R,-1)$ can be written as $[\delta,-]$, where $\delta \in \widehat{\operatorname{Pol}}(A / R,-1)^{1}$ is the element defined by the derivation $\delta$ on $A$.

Strictly speaking, $\widehat{\text { Pol }}$ is the complex of multiderivations, as polyvectors are usually defined as symmetric powers of the tangent complex. The two definitions agree (modulo completion) whenever the tangent complex is perfect, and Definition 1.4 is the more natural object when the definitions differ.

Definition 1.5. Define a decreasing filtration $F$ on $\widehat{\operatorname{Pol}}(A / R,-1)$ by

$$
F^{i} \widehat{\operatorname{Pol}}(A / R,-1):=\prod_{j \geqslant i} \underline{\operatorname{Hom}}_{A}\left(\operatorname{CoS}_{A}^{j} \Omega_{A / R}^{1}, A\right) \hbar^{j-1}
$$




\section{QUANTISING (-1)-SHIFTED SYMPLECTIC STRUCTURES}

this has the properties that $\widehat{\operatorname{Pol}}(A / R,-1)=\lim _{i} \widehat{\operatorname{Pol}}(A / R,-1) / F^{i}$, with $\left[F^{i}, F^{j}\right] \subset F^{i+j-1}$, $\delta F^{i} \subset F^{i}$, and $F^{i} F^{j} \subset \hbar^{-1} F^{i+j}$.

Observe that this filtration makes $F^{2} \widehat{\operatorname{Pol}}(A / R,-1)$ into a pro-nilpotent DGLA.

Definition 1.6. Define the tangent DGLA of polyvectors by

$$
T \widehat{\operatorname{Pol}}(A / R,-1):=\widehat{\operatorname{Pol}}(A / R,-1) \oplus \prod_{p \geqslant 0} \underline{\operatorname{Hom}}_{A}\left(\operatorname{CoS}_{A}^{p}\left(\Omega_{A / R}^{1}\right), A\right) \hbar^{p} \epsilon
$$

for $\epsilon$ of degree 0 with $\epsilon^{2}=0$. The Lie bracket is given by $[u+v \epsilon, x+y \epsilon]=[u, x]+[u, y] \epsilon+[v, x] \epsilon$.

Definition 1.7. Given a Maurer-Cartan element $\pi \in \operatorname{MC}\left(F^{2} \widehat{\operatorname{Pol}}(A / R,-1)\right)$, define

$$
T_{\pi} \widehat{\operatorname{Pol}}(A / R,-1):=\prod_{p \geqslant 0} \underline{\operatorname{Hom}}_{A}\left(\operatorname{CoS}_{A}^{p}\left(\Omega_{A / R}^{1}\right), A\right) \hbar^{p}
$$

with derivation $\delta+[\pi,-]$ (necessarily square-zero by the Maurer-Cartan conditions).

The product on polyvectors makes this a CDGA (with no need to rescale the product by $\hbar$ ), and it inherits the filtration $F$ from $\widehat{\mathrm{Pol}}$.

Given $\pi \in \operatorname{MC}\left(F^{2} \widehat{\operatorname{Pol}}(A / R,-1) / F^{p}\right)$, we define $T_{\pi} \widehat{\operatorname{Pol}}(A / R,-1) / F^{p}$ similarly. This is a CDGA because $F^{i} \cdot F^{j} \subset F^{i+j}$.

Regarding $T_{\pi} \widehat{\operatorname{Pol}}(A / R,-1)$ as an abelian DGLA, observe that $\operatorname{MC}\left(T_{\pi} \widehat{\operatorname{Pol}}(A / R,-1)\right)$ is just the fibre of $\operatorname{MC}(T \widehat{\operatorname{Pol}}(A / R,-1)) \rightarrow \operatorname{MC}(\widehat{\operatorname{Pol}}(A / R,-1))$ over $\pi$. Evaluation at $\hbar=1$ gives an isomorphism from $T \widehat{\operatorname{Pol}}(A / R,-1)$ to the $\operatorname{DGLA} \widehat{\operatorname{Pol}}(A / R,-1) \otimes_{\mathbb{Q}} \mathbb{Q}[\epsilon]$ of $[\operatorname{Pri17}, \S 1.1 .1]$, and the map $\sigma$ of [Pri17, Definition 1.11] then becomes the following.

Definition 1.8. Define

$$
\sigma=-\partial_{\hbar^{-1}}: \widehat{\operatorname{Pol}}(A / R,-1) \rightarrow T \widehat{\operatorname{Pol}}(A / R,-1)
$$

by $\alpha \mapsto \alpha+\epsilon \hbar^{2} \partial \alpha / \partial \hbar$. Note that this is a morphism of filtered DGLAs, so gives a map

$$
\operatorname{MC}\left(F^{2} \widehat{\operatorname{Pol}}(A / R,-1)\right) \rightarrow \operatorname{MC}\left(F^{2} T \widehat{\operatorname{Pol}}(A / R,-1)\right),
$$

with $\sigma(\pi) \in \mathrm{Z}^{1}\left(F^{2} T_{\pi} \widehat{\operatorname{Pol}}(A / R,-1)\right)$.

\subsubsection{Quantised (-1)-shifted polyvectors}

Definition 1.9. Define a strict line bundle over $A$ to be an $A$-module $M$ in cochain complexes such that $M^{\#}$ is a projective module of rank 1 over the graded-commutative algebra $A^{\#}$ underlying $A$. Given $b \in \mathrm{Z}^{1} A$, define $A_{b}$ to be the strict line bundle $(A, \delta+b)$.

(When $A$ has elements of positive degree, note that $M$ might not be cofibrant in the projective model structure, but this does not affect anything.)

Definition 1.10. Given a strict line bundle $M$ over $A$, define the complex of quantised $(-1)$ shifted polyvector fields on $M$ by

$$
Q \widehat{\operatorname{Pol}}(M,-1):=\prod_{p \geqslant 0} F_{p} \mathscr{D}_{A / R}(M) \hbar^{p-1} .
$$

Multiplication of differential operators gives us a product

$$
Q \widehat{\operatorname{Pol}}(M,-1) \times Q \widehat{\operatorname{Pol}}(M,-1) \rightarrow \hbar^{-1} Q \widehat{\operatorname{Pol}}(M,-1),
$$




\section{J. P. PRIDHAM}

but the associated commutator $[-,-]$ takes values in $Q \widehat{\operatorname{Pol}}(M,-1)$, so $Q \widehat{\operatorname{Pol}}(M,-1)$ is a DGLA over $R$.

Note that the differential $\delta$ on $Q \widehat{\operatorname{Pol}}(M,-1)$ can be written as $\left[\delta_{M},-\right]$, where $\delta_{M} \in F_{1} \mathscr{D}_{A / R}(M)^{1}$ is the element defined by the differential $\delta_{M}$ on $M$. In particular, $Q \widehat{\operatorname{Pol}}\left(A_{b},-1\right)$ is the gradedassociative algebra $Q \widehat{\operatorname{Pol}}(A,-1)$ with differential $\left[\delta_{A}+b,-\right]$.

Definition 1.11. Define a decreasing filtration $\tilde{F}$ on $Q \widehat{\operatorname{Pol}}(M,-1)$ by

$$
\tilde{F}^{i} Q \widehat{\operatorname{Pol}}(M,-1):=\prod_{j \geqslant i} F_{j} \mathscr{D}_{A / R}(M) \hbar^{j-1}
$$

this has the properties that $Q \widehat{\operatorname{Pol}}(M,-1)=\varliminf_{i} \widehat{\operatorname{Pol}}(M,-1) / \tilde{F}^{i}$, with $\left[\tilde{F}^{i}, \tilde{F}^{j}\right] \subset \tilde{F}^{i+j-1}, \delta \tilde{F}^{i} \subset \tilde{F}^{i}$, and $\tilde{F}^{i} \tilde{F}^{j} \subset \hbar^{-1} \tilde{F}^{i+j}$.

Observe that this filtration makes $\tilde{F}^{2} Q \widehat{\operatorname{Pol}}(M,-1)$ into a pro-nilpotent DGLA.

Definition 1.12. When $A$ is cofibrant, we define an $E_{0}$ quantisation of $M$ over $R$ to be a MaurerCartan element

$$
\Delta \in \operatorname{MC}\left(\tilde{F}^{2} Q \widehat{\operatorname{Pol}}(M,-1)\right) .
$$

The associated $R \llbracket \hbar \rrbracket$-module $M_{\Delta}$ is given by $M \llbracket \hbar \rrbracket$ equipped with the differential $\delta_{M}+\Delta$, which is necessarily square-zero by the Maurer-Cartan condition. We then have $M_{\Delta} /\left(\hbar M_{\Delta}\right)=M$, because $\hbar \mid \Delta$.

Remark 1.13. A more conceptual way to interpret such $E_{0}$ quantisations is as deformations of $M$ as a module over the de Rham pro-algebra $\operatorname{DR}(A / R)$. Such a deformation of $M$ is the same as a deformation of the right $\mathscr{D}_{A}$-module $M \otimes_{A} \mathscr{D}_{A}$, and a Maurer-Cartan element $\Delta$ gives a deformation $x \mapsto \delta(x)+\Delta \cdot x$ of the differential $\delta$ on $M \otimes_{A} \mathscr{D}_{A} \llbracket \hbar \rrbracket$.

Equivalently, a $(-1)$-shifted Poisson structure on $\mathscr{O}_{X}$ is the structure of an $\mathscr{O}_{X} \llbracket \Omega_{X}^{1}[-1] \rrbracket=$ $\hat{\mathscr{O}}_{T X[1]}$-module, and an $E_{0}$ quantisation is a lifting of such a structure making $\mathscr{O}_{X} \llbracket \hbar \rrbracket$ a module over the Rees pro-algebra $\mathscr{R}:=\prod_{p \in \mathbb{Z}} \hbar^{-p} F^{p} \mathrm{DR}(A / R)$, via the isomorphism $\mathscr{R} / \hbar \mathscr{R} \cong$ $\hat{\mathscr{O}}_{T X[1]}$.

In many ways, deformations over $\operatorname{DR}(A / R)$ are more natural than $R$-module deformations, because the de Rham algebra is the natural algebraic analogue of the analytic sheaf of complex constants. For $n>0$, the $E_{n}$-algebra deformations over $R$ and over $\operatorname{DR}(A / R)$ are the same by the Hochschild-Kostant-Rosenberg (HKR for short) isomorphism, but for $n=0$, the $\operatorname{DR}(A / R)$-module structure imposes the condition that deformations be given by differential operators.

Remark 1.14. Observe that when the $E_{0}$ quantisation $\Delta$ is linear in $\hbar$, it is a second-order differential operator. When $M=A$ and $\Delta(1)=0$, this gives exactly the structure of a $B V$ algebra over $R \llbracket \hbar \rrbracket$, the associated Lie bracket being given by the image of $\Delta$ in $\hbar\left(F_{2} \mathscr{D}_{A} / F_{1} \mathscr{D}_{A}\right) \cong$ $\hbar \operatorname{Symm}_{A}^{2} \mathscr{T}_{A}$. In general, for any $E_{0}$ quantisation $\Delta$ of $A$ with $\Delta(1)=0$, the pair $(A, \Delta)$ is a commutative $B V_{\infty}$-algebra in the sense of [Kra00, Definition 9].

Remark 1.15. For unbounded CDGAs, the hypothesis that $A$ be cofibrant seems unnecessarily strong. Most of our results will hold when $A^{\#}$ is free or even when $A$ is semi-smooth. This suggests that the most natural notion of equivalence for CDGAs in this setting might be not quasi-isomorphism but Morita equivalence of derived categories of the second kind (in the sense 


\section{QuANTISING (-1)-SHIFTED SYMPLECTIC STRUCTURES}

of [Pos11]). Dealing with semi-smooth CDGAs might provide an alternative approach to the stacky CDGAs featuring in Section 3 to study Artin stacks.

Definition 1.16. Given a DGLA L, define the Maurer-Cartan set by

$$
\operatorname{MC}(L):=\left\{\omega \in L^{1}: d \omega+\frac{1}{2}[\omega, \omega]=0 \in L^{2}\right\} .
$$

Following [Hin01], define the Maurer-Cartan space $\underline{\mathrm{MC}}(L)$ (a simplicial set) of a nilpotent DGLA $L$ by

$$
\underline{\operatorname{MC}}(L)_{n}:=\operatorname{MC}\left(L \otimes \mathbb{Q} \Omega^{\bullet}\left(\Delta^{n}\right)\right),
$$

where

$$
\Omega^{\bullet}\left(\Delta^{n}\right)=\mathbb{Q}\left[t_{0}, t_{1}, \ldots, t_{n}, \delta t_{0}, \delta t_{1}, \ldots, \delta t_{n}\right] /\left(\sum t_{i}-1, \sum \delta t_{i}\right)
$$

is the commutative dg algebra of de Rham polynomial forms on the $n$-simplex, with the $t_{i}$ of degree 0 .

Definition 1.17. We now define another decreasing filtration $G$ on $Q \widehat{\operatorname{Pol}}(M,-1)$ by setting

$$
G^{i} Q \widehat{\operatorname{Pol}}(M,-1):=\hbar^{i} Q \widehat{\operatorname{Pol}}(M,-1) \text {. }
$$

We then set $G^{i} \tilde{F}^{p}:=G^{i} \cap \tilde{F}^{p}$.

Note that $G^{i} \subset \tilde{F}^{i}$, and beware that $G^{i} \tilde{F}^{p}$ is not the same as $\hbar^{i} \tilde{F}^{p}$ in general since

$$
\begin{aligned}
G^{i} \tilde{F}^{p} Q \widehat{\operatorname{Pol}}(M,-1) & =\prod_{j \geqslant p} F_{j-i} \mathscr{D}_{A / R}(M) \hbar^{j-1}, \\
\hbar^{i} \tilde{F}^{p} Q \widehat{\operatorname{Pol}}(M,-1) & =\prod_{j \geqslant p+i} F_{j-i} \mathscr{D}_{A / R}(M) \hbar^{j-1} .
\end{aligned}
$$

We will also consider the convolution $G * \tilde{F}$ given by $(G * \tilde{F})^{p}:=\sum_{i+j=p} G^{i} \cap \tilde{F}^{j}$; explicitly,

$$
(G * \tilde{F})^{p} Q \widehat{\operatorname{Pol}}(M,-1)=\prod_{j<p} F_{2 j-p} \mathscr{D}_{A / R}(M) \hbar^{j-1} \times \prod_{j \geqslant p} F_{j} \mathscr{D}_{A / R}(M) \hbar^{j-1} .
$$

In particular, $(G * \tilde{F})^{2} Q \widehat{\operatorname{Pol}}(M,-1)=A \oplus \tilde{F}^{2} Q \widehat{\operatorname{Pol}}(M,-1)$.

Definition 1.18. Define the space $Q \mathcal{P}(M,-1)$ of $E_{0}$ quantisations of $M$ over $R$ to be given by the simplicial set

$$
Q \mathcal{P}(M,-1):=\underbrace{}_{i} \underline{\mathrm{MC}}\left(\tilde{F}^{2} Q \widehat{\operatorname{Pol}}(M,-1) / \tilde{F}^{i+2}\right)
$$

Also write

$$
Q \mathcal{P}(M,-1) / G^{k}:=\varliminf_{i} \underline{\operatorname{MC}}\left(\tilde{F}^{2} Q \widehat{\operatorname{Pol}}(M,-1) /\left(\tilde{F}^{i+2}+G^{k}\right)\right),
$$

so $Q \mathcal{P}(M,-1)=\lim _{k} Q \mathcal{P}(M,-1) / G^{k}$.

We will also consider twisted quantisations

$$
Q^{\mathrm{tw}} \mathcal{P}(M,-1):={\underset{\varliminf}{i}}_{\lim _{i}} \underline{\mathrm{MC}}\left((G * \tilde{F})^{2} Q \widehat{\mathrm{Pol}}(M,-1) / \tilde{F}^{i+2}\right)
$$

these are just quantisations of strict line bundles $M \otimes_{A} A_{b}$ for $b \in \mathrm{Z}^{1}(A)$. 


\section{J. P. PRIDHAM}

\subsubsection{The centre of a quantisation}

DeFinition 1.19. Define the filtered tangent DGLA of quantised polyvectors by

$$
\begin{gathered}
T Q \widehat{\operatorname{Pol}}(M,-1):=Q \widehat{\operatorname{Pol}}(M,-1) \oplus \prod_{p \geqslant 0} F_{p} \mathscr{D}_{A / R}(M) \hbar^{p} \epsilon, \\
\tilde{F}^{j} T Q \widehat{\operatorname{Pol}}(M,-1):=\tilde{F}^{j} Q \widehat{\operatorname{Pol}}(M,-1) \oplus \prod_{p \geqslant j} F_{p} \mathscr{D}_{A / R}(M) \hbar^{p} \epsilon
\end{gathered}
$$

for $\epsilon$ of degree 0 with $\epsilon^{2}=0$. The Lie bracket is given by $[u+v \epsilon, x+y \epsilon]=[u, x]+[u, y] \epsilon+[v, x] \epsilon$.

Definition 1.20. Given a Maurer-Cartan element $\Delta \in \operatorname{MC}\left(\tilde{F}^{2} Q \widehat{\operatorname{Pol}}(M,-1)\right)$, define the centre of $(M, \Delta)$ by

$$
T_{\Delta} Q \widehat{\operatorname{Pol}}(M,-1):=\prod_{p \geqslant 0} F_{p} \mathscr{D}_{A / R}(M) \hbar^{p},
$$

with derivation $\delta+[\Delta,-]$ (necessarily square-zero by the Maurer-Cartan conditions).

Multiplication of differential operators makes this a DGAA (with no need to rescale the product by $\hbar)$, and it has a filtration

$$
\tilde{F}^{i} T_{\Delta} Q \widehat{\operatorname{Pol}}(M,-1):=\prod_{p \geqslant i} F_{p} \mathscr{D}_{A / R}(M) \hbar^{p},
$$

with $\tilde{F}^{i} \cdot \tilde{F}^{j} \subset \tilde{F}^{i+j}$. Given $\Delta \in \operatorname{MC}\left(F^{2} Q \widehat{\operatorname{Pol}}(M,-1) / \tilde{F}^{p}\right)$, we define $T_{\Delta} Q \widehat{\operatorname{Pol}}(M,-1) / \tilde{F}^{p}$ similarly - this is also a DGAA as $\tilde{F}^{p}$ is an ideal.

Observe that $T_{\Delta} Q \mathcal{P}(M,-1):=\underline{\mathrm{MC}}\left(\tilde{F}^{2} T_{\Delta} Q \widehat{\mathrm{Pol}}(M,-1)\right)$ is just the fibre of

$$
\underline{\mathrm{MC}}\left(\tilde{F}^{2} T Q \widehat{\operatorname{Pol}}(M,-1)\right) \rightarrow \underline{\mathrm{MC}}\left(\tilde{F}^{2} Q \widehat{\operatorname{Pol}}(M,-1)\right) \text { over } \Delta .
$$

Similarly to Definition 1.17 , there are filtrations on $T Q \widehat{\operatorname{Pol}}(M,-1)$ and $T_{\Delta} Q \widehat{\operatorname{Pol}}(M,-1)$ given by powers of $\hbar$; we denote both by $G$. Since $\operatorname{gr}_{G}^{i} \tilde{F}^{p-i} Q \widehat{\mathrm{Pol}}=\prod_{j \geqslant p-i} \operatorname{gr}_{j-i}^{F} \mathscr{D}_{A / R}(M) \hbar^{j-1}$, the associated graded pieces of the filtration admit maps

$$
\begin{gathered}
\operatorname{gr}_{G}^{i} \tilde{F}^{p} Q \widehat{\operatorname{Pol}}(M,-1) \rightarrow \prod_{j \geqslant p} \underline{\operatorname{Hom}_{A}}\left(\operatorname{CoS}_{A}^{j-i}\left(\Omega_{A / R}^{1}\right) \otimes_{A} M, M\right) \hbar^{j-1}, \\
\operatorname{gr}_{G}^{i} \tilde{F}^{p} T_{\Delta} Q \widehat{\operatorname{Pol}}(M,-1) \rightarrow \prod_{j \geqslant p} \underline{\operatorname{Hom}}_{A}\left(\operatorname{CoS}_{A}^{j-i}\left(\Omega_{A / R}^{1}\right) \otimes_{A} M, M\right) \hbar^{j}
\end{gathered}
$$

which are isomorphisms when $A$ is semi-smooth (in particular, whenever $A$ is cofibrant as a CDGA over $R$ ).

For the filtration $F$ of Definition 1.5, we may rewrite these maps as

$$
\begin{aligned}
\operatorname{gr}_{G}^{i} \tilde{F}^{p} Q \widehat{\operatorname{Pol}}(A,-1) & \rightarrow F^{p-i} \widehat{\operatorname{Pol}}(A,-1) \hbar^{i}, \\
\operatorname{gr}_{G}^{i} \tilde{F}^{p} T_{\Delta} Q \widehat{\operatorname{Pol}}(M,-1) & \rightarrow F^{p-i} T_{\pi_{\Delta}} \widehat{\operatorname{Pol}}(A,-1) \hbar^{i},
\end{aligned}
$$

where $\pi_{\Delta} \in \operatorname{MC}\left(F^{2} \widehat{\operatorname{Pol}}(A,-1)\right)$ denotes the image of $\Delta$ under the $\operatorname{map} \operatorname{gr}_{G}^{0} \tilde{F}^{2} Q \widehat{\operatorname{Pol}}(A,-1) \rightarrow$ $F^{2} \widehat{\operatorname{Pol}}(A,-1)$.

Since the cohomology groups of $T_{\pi_{\Delta}} \widehat{\mathrm{Pol}}(A,-1)$ are Poisson cohomology, we will refer to the cohomology groups of $T_{\Delta} Q \widehat{\mathrm{Pol}}(M,-1)$ as quantised Poisson cohomology.

We write $T_{\Delta} Q^{\mathrm{tw}} \mathcal{P}(M,-1):=\underline{\mathrm{MC}}\left((G * \tilde{F})^{2} T_{\Delta} Q \widehat{\operatorname{Pol}}(M,-1)\right)$. 


\section{QuANTISING (-1)-SHIFTED SYMPLECTIC STRUCTURES}

Definition 1.21. Say that an $E_{0}$ quantisation $\Delta=\sum_{j \geqslant 2} \Delta_{j} \hbar^{j}$ is non-degenerate if the map

$$
\Delta_{2}^{\sharp}: M \otimes_{A} \Omega_{A}^{1} \rightarrow \underline{\operatorname{Hom}}_{A}\left(\Omega_{A}^{1}, M\right)[1]
$$

is a quasi-isomorphism and $\Omega_{A}^{1}$ is perfect.

Definition 1.22. Define the tangent spaces

$$
\begin{aligned}
& T Q \mathcal{P}(M,-1):={\underset{\iota}{i}}_{\lim } \underline{\mathrm{MC}}\left(\tilde{F}^{2} T Q \widehat{\operatorname{Pol}}(M,-1) / \tilde{F}^{i+2}\right), \\
& T Q^{\mathrm{tw}} \mathcal{P}(M,-1):={\underset{\leftarrow}{i}}_{\lim } \underline{\mathrm{MC}}\left((G * \tilde{F})^{2} T Q \widehat{\operatorname{Pol}}(M,-1) / \tilde{F}^{i+2}\right),
\end{aligned}
$$

with $T Q \mathcal{P}(M,-1) / G^{k}$ and $T Q^{\mathrm{tw}} \mathcal{P}(M,-1) / G^{k}$ defined similarly.

These are simplicial sets over $Q \mathcal{P}(M,-1)\left(Q^{\mathrm{tw}} \mathcal{P}(M,-1), Q \mathcal{P}(M,-1) / G^{k}, Q^{\mathrm{tw}} \mathcal{P}(M,-1) / G^{k}\right.$, respectively), fibred in simplicial abelian groups.

Definition 1.23. Define the canonical tangent vector

$$
\sigma=-\partial_{\hbar^{-1}}: Q \widehat{\operatorname{Pol}}(M,-1) \rightarrow T Q \widehat{\operatorname{Pol}}(M,-1)
$$

by $\alpha \mapsto \alpha+\epsilon \hbar^{2} \partial \alpha / \partial \hbar$. Note that this is a morphism of filtered DGLAs, so gives a map $\sigma: Q \mathcal{P}(M,-1) \rightarrow T Q \mathcal{P}(M,-1)$ with $\sigma(\Delta) \in \mathrm{Z}^{1}\left(\tilde{F}^{2} T_{\Delta} Q \widehat{\operatorname{Pol}}(M,-1)\right)$.

1.2. Generalised pre-symplectic structures. We now develop generalised shifted pre-symplectic structures as certain power series in the de Rham complex, leading to a notion of compatibility between these generalised structures and quantisations.

Definition 1.24. Define the de Rham complex $\mathrm{DR}(A / R)$ to be the product total complex of the bicomplex

$$
A \stackrel{d}{\rightarrow} \Omega_{A / R}^{1} \stackrel{d}{\rightarrow} \Omega_{A / R}^{2} \stackrel{d}{\rightarrow} \cdots,
$$

so the total differential is $d \pm \delta$.

We define the Hodge filtration $F$ on $\operatorname{DR}(A / R)$ by setting $F^{p} \operatorname{DR}(A / R) \subset \mathrm{DR}(A / R)$ to consist of terms $\Omega_{A / R}^{i}$ with $i \geqslant p$. In particular, $F^{p} \operatorname{DR}(A / R)=\operatorname{DR}(A / R)$ for $p \leqslant 0$.

Definition 1.25. When $A$ is a cofibrant CDGA over $R$, recall that a (-1)-shifted pre-symplectic structure $\omega$ on $A / R$ is an element $\omega \in \mathrm{Z}^{1} F^{2} \mathrm{DR}(A / R)$. In [PTVV13], shifted pre-symplectic structures are referred to as closed 2-forms.

A (-1)-shifted pre-symplectic structure $\omega$ is called symplectic if $\omega_{2} \in \mathrm{Z}^{-1} \Omega_{A / R}^{2}$ induces a quasi-isomorphism

$$
\omega_{2}^{\sharp}: \operatorname{Hom}_{A}\left(\Omega_{A / R}^{1}, A\right) \rightarrow \Omega_{A / R}^{1}[-1],
$$

and $\Omega_{A / R}^{1}$ is perfect as an $A$-module.

In order to define compatibility functors for quantisations, we will need to construct $A_{\infty^{-}}$ morphisms from the de Rham algebra, which we will do using the following DGAA resolution.

Definition 1.26. Write $A^{\otimes \bullet+1}$ for the cosimplicial CDGA $n \mapsto A^{n+1}$ given by the Cech nerve, with $I$ the kernel of the diagonal map $A^{\otimes \bullet+1} \rightarrow A$. This has a filtration $F$ given by powers $F^{p}:=(I)^{p}$ of $I$, and we define the filtered cosimplicial CDGA $\hat{A}^{\otimes \bullet+1}$ to be the completion

$$
\hat{A}^{\otimes \bullet+1}:=\lim _{q} A^{\otimes \bullet+1} / F^{q}, \quad F^{p} \hat{A}^{\otimes \bullet+1}:=\underbrace{\lim }_{q} F^{p} / F^{q} .
$$




\section{J. P. PRIDHAM}

We then take the Dold-Kan conormalisation $N \hat{A}^{\bullet+1}$, which becomes a filtered bi-DGAA via the Alexander-Whitney cup product. Explicitly, $N^{n} \hat{A}^{\bullet+1}$ is the intersection of the kernels of all the big diagonals $\hat{A}^{n+1} \rightarrow \hat{A}^{n}$, and the cup product is given by

$$
\left(a_{0} \otimes \cdots \otimes a_{m}\right) \smile\left(b_{0} \otimes \cdots \otimes b_{n}\right)=a_{0} \otimes \cdots \otimes a_{m-1} \otimes\left(a_{m} b_{0}\right) \otimes b_{1} \otimes \cdots \otimes b_{n} .
$$

We then define $\operatorname{DR}^{\prime}(A / R)$ to be the product total complex

$$
\operatorname{DR}^{\prime}(A / R):=\operatorname{Tot}^{\Pi} N \hat{A}^{\bullet+1}
$$

regarded as a filtered DGAA over $R$, with $F^{p} \operatorname{DR}^{\prime}(A / R):=\operatorname{Tot}^{\Pi} N F^{p} \hat{A}^{\bullet+1}$.

The following is standard.

LEMma 1.27. There is a filtered quasi-isomorphism $\operatorname{DR}^{\prime}(A / R) \rightarrow \operatorname{DR}(A / R)$ given by $N^{n} \hat{A}^{\bullet+1} \rightarrow$ $N^{n} \hat{A}^{\bullet+1} / F^{n+1} \cong\left(\Omega_{A / R}^{1}\right)^{\otimes_{A} n} \rightarrow \Omega_{A / R}^{n}$.

Proof. It suffices to show that the map is a quasi-isomorphism on the graded pieces associated to the filtration. Now, $\operatorname{gr}_{F}^{p} \hat{A}^{\otimes \bullet+1}=\operatorname{Symm}_{A}^{p} D\left(\Omega_{A}^{1}[-1]\right)$, where $D$ denotes Dold-Kan denormalisation from cochain complexes to cosimplicial complexes. Thus

$$
\operatorname{Ngr}_{F}^{p} \hat{A}^{\otimes \bullet+1}=N \operatorname{Symm}_{A}^{p} D\left(\Omega_{A}^{1}[-1]\right),
$$

so $\operatorname{Tot}^{\Pi} N \operatorname{Symm}_{A}^{p} D\left(\Omega_{A}^{1}[-1]\right)$ is quasi-isomorphic to $\operatorname{Symm}_{A}^{p} N D\left(\Omega_{A}^{1}[-1]\right)=\operatorname{Symm}_{A}^{p}\left(\Omega_{A}^{1}[-1]\right)=$ $\Omega_{A}^{p}[-p]$, combining the Dold-Kan denormalisation with the Eilenberg-Zilber shuffle map.

DeFinition 1.28. Define a decreasing filtration $\tilde{F}$ on $\operatorname{DR}^{\prime}(A / R) \llbracket \hbar \rrbracket$ by

$$
\tilde{F}^{p} \operatorname{DR}^{\prime}(A / R):=\prod_{i \geqslant 0} F^{p-i} \operatorname{DR}^{\prime}(A / R) \hbar^{i}
$$

where we adopt the convention that $F^{j} \mathrm{DR}^{\prime}=\mathrm{DR}^{\prime}$ for all $j \leqslant 0$.

Define further filtrations $G$ and $G * \tilde{F}$ by $G^{k} \operatorname{DR}^{\prime}(A / R) \llbracket \hbar \rrbracket=\hbar^{k} \operatorname{DR}^{\prime}(A / R) \llbracket \hbar \rrbracket$ and $(G * \tilde{F})^{p}:=$ $\sum_{i+j=p} G^{i} \cap \tilde{F}^{j}$, so

$$
(G * \tilde{F})^{p}=\prod_{i \geqslant 0} F^{p-2 i} \mathrm{DR}^{\prime}(A / R) \hbar^{i}
$$

This makes $\left(\mathrm{DR}^{\prime}(A / R) \llbracket \hbar \rrbracket, G * \tilde{F}\right)$ into a filtered DGAA since $\tilde{F}^{p} \tilde{F}^{q} \subset \tilde{F}^{p+q}$ and similarly for $G$.

Definition 1.29. Define a generalised (-1)-shifted pre-symplectic structure on a cofibrant CDGA $A / R$ to be an element

$$
\omega \in \mathrm{Z}^{1}\left((G * \tilde{F})^{2} \mathrm{DR}^{\prime}(A / R) \llbracket \hbar \rrbracket\right)=\mathrm{Z}^{1}\left(F^{2} \mathrm{DR}^{\prime}(A / R)\right) \times \hbar \mathrm{Z}^{1} \mathrm{DR}^{\prime}(A / R) \llbracket \hbar \rrbracket .
$$

Call this symplectic if $\Omega_{A / R}^{1}$ is perfect as an $A$-module and the leading term $\omega_{0} \in \mathrm{Z}^{1} F^{2} \mathrm{DR}^{\prime}(A / R)$ induces a quasi-isomorphism

$$
\left[\omega_{0}\right]^{\sharp}: \operatorname{Hom}_{A}\left(\Omega_{A / R}^{1}, A\right) \rightarrow \Omega_{A / R}^{1}[-1]
$$

for $\left[\omega_{0}\right] \in \mathrm{Z}^{-1} \Omega_{A / R}^{2}$ the image of $\omega_{0}$ modulo $F^{3}$.

DeFinition 1.30. Define the space of generalised (-1)-shifted pre-symplectic structures on $A / R$ to be the simplicial set

$$
G \operatorname{PreSp}(A / R,-1):=\underbrace{\lim }_{i} \underline{\mathrm{MC}}\left((G * \tilde{F})^{2} \mathrm{DR}^{\prime}(A / R) \llbracket \hbar \rrbracket / \tilde{F}^{i+2}\right),
$$




\section{QuANTISING (-1)-SHIFTED SYMPLECTIC STRUCTURES}

where we regard the cochain complex $\operatorname{DR}^{\prime}(A / R)$ as a DGLA with trivial bracket. Write PreSp $=$ $G \operatorname{PreSp} / G^{1}$.

Also write $G \operatorname{PreSp}(A / R,-1) / \hbar^{k}:=\lim _{i} \underline{\operatorname{MC}}\left((G * \tilde{F})^{2} \operatorname{DR}^{\prime}(A / R)[\hbar] /\left(G^{k}+\tilde{F}^{i+2}\right)\right)$, so $G \operatorname{PreSp}(A / R,-1)=\varliminf_{k} G \operatorname{PreSp}(A / R,-1) / \hbar^{k}$.

Set $G \operatorname{Sp}(A / R,-1) \subset G \operatorname{PreSp}(A / R,-1)$ to consist of the symplectic structures; this is a union of path-components.

Note that $G \operatorname{PreSp}(A / R,-1)$ is canonically weakly equivalent to the Dold-Kan denormalisation of the good truncation complex $\tau^{\leqslant 0}\left((G * \tilde{F})^{2} \operatorname{DR}(A / R) \llbracket \hbar \rrbracket[1]\right)$ (and similarly for the various quotients we consider), but the description in terms of $\underline{\mathrm{MC}}$ will simplify comparisons. In particular, we have

$$
\pi_{i} G \operatorname{PreSp}(A / R,-1) \cong \mathrm{H}^{1-i}\left(F^{2} \mathrm{DR}(A / R)\right) \times \hbar \mathrm{H}^{1-i}(\mathrm{DR}(A / R)) \llbracket \hbar \rrbracket .
$$

1.2.1. Compatible quantisations. We will now develop the notion of compatibility between a (truncated) generalised pre-symplectic structure and a (truncated) $E_{0}$ quantisation. For the 0th order truncation (corresponding to $k=1$ in Definition 1.36), this recovers the notion of compatibility between pre-symplectic and Poisson structures from [Pri17].

LEMma 1.31. Take a complete filtered graded-associative $R$-algebra $\left(B\right.$, Fil $\left.^{\bullet}\right)$ and a morphism $\phi: A^{\#} \rightarrow \mathrm{Fil}^{0} B$ of graded $R$-algebras; assume that the left and right $A$-module structures on $\operatorname{gr}_{\mathrm{Fil}} B$ agree. Then for any $\Delta \in \mathrm{Fil}^{0} B^{1}$, there is an associated morphism

$$
\mu(-, \Delta):\left(\mathrm{DR}^{\prime}(A)^{\#}, F^{\bullet}\right) \rightarrow\left(B, \mathrm{Fil}^{\bullet}\right)
$$

of filtered graded-associative $R$-algebras induced by the graded algebra map on $A^{\otimes \bullet+1}$ determined by $\mu(1 \otimes 1, \Delta)=\Delta$ and $\mu(a, \Delta)=a$ for $a \in A$.

Given $\rho \in \mathrm{Fil}^{j} B^{k}$, there is then a filtered $R$-linear derivation

$$
\nu(-, \Delta, \rho):\left(\mathrm{DR}^{\prime}(A / R)^{\#}, F^{\bullet}\right) \rightarrow\left(B[k], \mathrm{Fil}^{\bullet+j}\right)
$$

induced by the $\mu(-, \Delta)$-derivation on $A^{\otimes \bullet+1}$ determined by $\nu(1 \otimes 1, \Delta, \rho)=\rho$.

Proof. Explicitly, $\mu(-, \Delta)$ is given on $A^{\otimes \bullet+1}$ by

$$
\left(a_{0} \otimes a_{1} \otimes \cdots \otimes a_{r}\right) \mapsto a_{0} \Delta a_{1} \Delta \cdots \Delta a_{r}
$$

because $a \otimes b=a \smile(1 \otimes 1) \smile b$ and so on. Similarly, $\nu(-, \Delta, \rho)$ is given by

$$
\left(a_{0} \otimes a_{1} \otimes \cdots \otimes a_{r}\right) \mapsto \sum_{i=0}^{r-1} \pm a_{0} \Delta a_{1} \Delta \cdots \Delta a_{i} \rho a_{i+1} \Delta \cdots \Delta a_{r} .
$$

We need to show that these respect the filtrations, so give maps on $\hat{A}^{\otimes \bullet+1}$ and hence filtered morphisms on $\operatorname{DR}(A / R)^{\prime}$.

Observe that the filtration on $A^{\otimes \bullet+1}$ is generated by that on $A^{\otimes 2}$, in the sense that

$$
F^{p} A^{\otimes m+1}=\sum_{p_{1}+\cdots+p_{m}=p}\left(F^{p_{1}} A^{\otimes 2}\right) \smile \cdots \smile\left(F^{p_{m}} A^{\otimes 2}\right) .
$$

It therefore suffices to show that $\mu(-, \Delta): A^{\otimes 2} \rightarrow B$ and $\nu(-, \Delta, \rho)$ are appropriately filtered.

Writing $[x, y]:=x \smile y-(-1)^{\operatorname{deg} x \operatorname{deg} y} y \smile x$ and $\cdot$ for the internal multiplication on $A^{\otimes r}$, it follows that for $a \in A$ and $x \in A \otimes A$, we have $[a, x]=(a \otimes 1 \mp 1 \otimes a) \cdot x$. Since $I=\operatorname{ker}\left(A^{\otimes 2} \rightarrow A\right)$ 


\section{J. P. PRIDHAM}

is generated by elements of the form $(a \otimes 1 \mp 1 \otimes a)$, this means that $[A, J]=I \cdot J$ for all ideals $J$ in $A^{\otimes 2}$. Because $F^{p}=I^{\cdot p}$, this gives

$$
F^{p} A^{\otimes 2}=\underbrace{[A,[A, \ldots[A,}_{p} A^{\otimes 2}] \ldots],
$$

so $F$ is the smallest multiplicative filtration for which left and right $A$-modules structures on $\operatorname{gr}_{F} A^{\otimes \bullet+1}$ agree.

Therefore the algebra homomorphism $\mu(-, \Delta)$ must send $F^{p}$ to $\mathrm{Fil}^{p}$, and the derivation $\nu(-$, $\Delta, \rho)$ must send $F^{p}$ to $\mathrm{Fil}^{p+j}$; in particular, the maps descend to the completion $\mathrm{DR}^{\prime}(A / R)$.

Lemma 1.32. Given $\Delta \in\left((G * \tilde{F})^{2} Q \widehat{\operatorname{Pol}}(M,-1) / G^{k}\right)^{1}$, Lemma 1.31 gives a morphism

$$
\mu(-, \Delta): \operatorname{DR}^{\prime}(A / R)[\hbar] / G^{k} \rightarrow T_{\Delta} Q \widehat{\operatorname{Pol}}(M,-1) / G^{k}
$$

of graded-associative $R[\hbar] / \hbar^{k}$-algebras respecting the filtrations $(G * \tilde{F})$.

Given $\rho \in\left((G * \tilde{F})^{p} T_{\Delta} Q \widehat{\operatorname{Pol}}(M,-1) / G^{k}\right)^{r}$, there is then a $R[\hbar] / \hbar^{k}$-linear derivation

$$
\nu(-, \Delta, \rho):\left(\mathrm{DR}^{\prime}(A / R)[\hbar] / \hbar^{k},(G * \tilde{F})^{\bullet}\right) \rightarrow\left(T_{\Delta} Q \widehat{\operatorname{Pol}}(M,-1)[r] / G^{k},(G * \tilde{F})^{\bullet+p}\right) .
$$

Proof. It suffices to prove this for the limit over all $k$, as $\Delta$ and $\rho$ always lift to $(G * \tilde{F})^{2} Q \widehat{\operatorname{Pol}}(M,-1)$. Set $T=T_{0} Q \widehat{\operatorname{Pol}}(M,-1)\left[\hbar^{-1}\right]$, with filtrations $\tilde{F}$ given by powers of $\hbar$ and $G^{i} T:=\hbar^{i} T_{0} Q \widehat{\operatorname{Pol}}(M,-1)$, so $G^{i} \tilde{F}^{j} T=\prod_{p \geqslant j} \hbar^{p} F_{p-i}$. These filtrations are multiplicative, with $\left[G^{i}, G^{j}\right] \subset G^{i+j-1}$, so the convolution filtration also satisfies $\left[(G * \tilde{F})^{i},(G * \tilde{F})^{j}\right] \subset(G * \tilde{F})^{i+j-1}$; in particular, $\operatorname{gr}_{G * \tilde{F}} T$ is commutative, so its left and right $A$-module structures agree (the same is not true of $\operatorname{gr}_{\tilde{F}} T$, which makes the convolution filtration necessary). Explicitly, $(G * \tilde{F})^{p} T=\prod_{k} \hbar^{k} F_{2 k-p}$.

Then $Q \widehat{\operatorname{Pol}}(M,-1)=G^{-1} T$ and $T_{0} Q \widehat{\operatorname{Pol}}(M,-1)=G^{0} T$, with $G^{i} \tilde{F}^{j} T=G^{i} \tilde{F}^{j} T_{0} Q \widehat{\operatorname{Pol}}(M,-1)$ and $G^{i-1} \tilde{F}^{j-1} T=G^{i} \tilde{F}^{j} Q \widehat{\operatorname{Pol}}(M,-1)$ whenever $i \geqslant 0$. Thus $(G * \tilde{F})^{p} Q \widehat{\operatorname{Pol}} \subset(G * \tilde{F})^{p-2} T$, so in particular $\Delta$ lies in $(G * \tilde{F})^{0} T$. Lemma 1.31 now gives filtered morphisms

$$
\begin{gathered}
\mu(-, \Delta):\left(\operatorname{DR}^{\prime}(A / R), F^{\bullet}\right) \rightarrow\left(T,(G * \tilde{F})^{\bullet}\right), \\
\nu(-, \Delta, \rho):\left(\operatorname{DR}^{\prime}(A / R), F^{\bullet}\right) \rightarrow\left(T[r],(G * \tilde{F})^{\bullet+p}\right) .
\end{gathered}
$$

Since $\Delta \in G^{-1} T$, we have $[\Delta, a] \in G^{0} T$ for all $a \in A$, so $\mu\left(F^{1}(A \otimes A), \Delta\right) \subset G^{0} T$. Since $F^{1}(A \otimes A)$ topologically generates $\operatorname{DR}^{\prime}(A / R)$ under multiplication, $\mu\left(\mathrm{DR}^{\prime}(A / R), \Delta\right)$ is thus contained in the subalgebra $G^{0} T=T_{0} Q \widehat{\operatorname{Pol}}(M,-1)$ of $T$. Extending linearly gives a map from $\operatorname{DR}^{\prime}(A / R) \llbracket \hbar \rrbracket$; since $\hbar G^{i} \tilde{F}^{q} T \subset G^{i+1} \tilde{F}^{q+1} T$, we then see that $\mu\left(\hbar^{i} F^{p-2 i}, \Delta\right) \subset G^{p-r-i} \tilde{F}^{r+i}$, so

$$
\mu\left((G * \tilde{F})^{p} \operatorname{DR}(A / R)^{\prime} \llbracket \hbar \rrbracket, \Delta\right) \subset(G * \tilde{F})^{p} T_{\Delta} Q \widehat{\operatorname{Pol}}(M,-1),
$$

and similarly for $\nu$.

LEMma 1.33. Take a complete filtered $R$-DGAA (B, Fil $\left.{ }^{\bullet}\right)$ and a morphism $\phi: A \rightarrow \mathrm{Fil}^{0} B$ of $R$-DGAAs; assume that the left and right $A$-module structures on $\operatorname{gr}_{\mathrm{Fil}} B$ agree. Then for any $\Delta \in \mathrm{Fil}^{0} B^{1}$ and any $\omega \in \mathrm{DR}^{\prime}(A / R)$, we have

$$
\begin{aligned}
{[\Delta, \mu(\omega, \Delta)] } & =\mu(d \omega, \Delta)+\frac{1}{2} \nu(\omega, \Delta,[\Delta, \Delta]), \\
\delta_{\Delta} \mu(\omega, \Delta) & =\mu(D \omega, \Delta)+\nu(\omega, \Delta, \kappa(\Delta)),
\end{aligned}
$$

where the $\delta$ are the structural differentials on $A$ and $B$, with $\delta_{\Delta}:=\delta+[\Delta,-], D:=d \pm \delta$ the total differential on $\mathrm{DR}^{\prime}(A / R)$, and $\kappa(\Delta):=[\delta, \Delta]+\frac{1}{2}[\Delta, \Delta]$. 


\section{QuANTISING (-1)-SHIFTED SYMPLECTIC STRUCTURES}

Proof. Both $[\Delta, \mu(-, \Delta)]$ and $\delta_{\Delta} \mu(-, \Delta)$ are derivations with respect to $\mu(-, \Delta)$, so it suffices to verify these identities on the generators $a$ and $d f$ of the denormalisation of $\operatorname{DR}^{\prime}(A / R)$, for $a, f \in A$.

In these cases, we have

$$
\begin{gathered}
{[\Delta, \mu(a, \Delta)]=[\Delta, a]=\mu(1 \otimes a \mp a \otimes 1, \Delta)=\mu(d a, \Delta),} \\
{[\Delta, \mu(1 \otimes f-f \otimes 1, \Delta)]=\Delta^{2} f \mp f \Delta^{2}=\frac{1}{2} \nu(d f, \Delta,[\Delta, \Delta]) .}
\end{gathered}
$$

Because $\nu(a, \Delta,[\Delta, \Delta])=0$ ( $\nu$ being $A$-linear $)$ and $d d f=0$, this gives the required results, the second set of equalities following by adding $\delta$.

In particular, Lemma 1.33 implies that when $\Delta \in \operatorname{MC}\left((G * \tilde{F})^{2} Q \widehat{\operatorname{Pol}}(A, n) / G^{k}\right)$ is an $E_{0}$ quantisation, $\mu(-, \Delta)$ is a chain map $\left(\right.$ since $\left.T_{\Delta} Q \widehat{\operatorname{Pol}}(M,-1)=\left(T_{0} Q \widehat{\operatorname{Pol}}(M,-1), \delta_{\Delta}\right)\right)$, so $\mu(-, \Delta)$ defines a map from de Rham cohomology to quantised Poisson cohomology.

DEFinition 1.34. We say that a generalised (-1)-shifted pre-symplectic structure $\omega$ and an $E_{0}$ quantisation $\Delta$ of a strict line bundle $M$ are compatible (or a compatible pair) if

$$
[\mu(\omega, \Delta)]=\left[-\partial_{\hbar^{-1}}(\Delta)\right] \in \mathrm{H}^{1}\left((G * \tilde{F})^{2} T_{\Delta} Q \widehat{\operatorname{Pol}}(M,-1)\right),
$$

where $\sigma=-\partial_{\hbar^{-1}}$ is the canonical tangent vector of Definition 1.23.

Definition 1.35. Given a simplicial set $Z$, an abelian group object $A$ in simplicial sets over $Z$, a space $X$ over $Z$, and a morphism $s: X \rightarrow A$ over $Z$, define the homotopy vanishing locus of $s$ over $Z$ to be the homotopy limit of the diagram

$$
X \underset{0}{\stackrel{s}{\longrightarrow}} A \longrightarrow Z \text {. }
$$

Definition 1.36. Define the space $Q \operatorname{Comp}(M,-1)$ of compatible quantised $(-1)$-shifted pairs to be the homotopy vanishing locus of

$$
(\mu-\sigma): G \operatorname{PreSp}(A / R,-1) \times Q \mathcal{P}(M,-1) \rightarrow T Q^{\mathrm{tw}} \mathcal{P}(M,-1)
$$

over $Q^{\mathrm{tw}} \mathcal{P}(M,-1)$

We define a cofiltration on this space by setting $Q \operatorname{Comp}(M,-1) / G^{k}$ to be the homotopy vanishing locus of

$$
(\mu-\sigma):\left(G \operatorname{PreSp}(A / R,-1) / G^{k}\right) \times\left(Q \mathcal{P}(M,-1) / G^{k}\right) \rightarrow T Q^{\mathrm{tw}} \mathcal{P}(M,-1) / G^{k}
$$

over $Q^{\operatorname{tw}} \mathcal{P}(M,-1) / G^{k}$.

When $k=1$, note that this recovers the notion of compatible $(-1)$-shifted pairs from [Pri17]. Definition 1.37. Define $Q \operatorname{Comp}(M,-1)^{\text {nondeg }} \subset Q \operatorname{Comp}(M,-1)$ to consist of compatible quantised pairs $(\omega, \Delta)$ with $\Delta$ non-degenerate. This is a union of path-components, and by [Pri17, Lemma 1.22] has a natural map $Q \operatorname{Comp}(M,-1)^{\text {nondeg }} \rightarrow G \operatorname{Sp}(A / R,-1)$ as well as the canonical $\operatorname{map} Q \operatorname{Comp}(M,-1)^{\text {nondeg }} \rightarrow Q \mathcal{P}(M,-1)^{\text {nondeg }}$.

Proposition 1.38. For any strict line bundle $M$, the canonical map

$$
Q \operatorname{Comp}(M,-1)^{\text {nondeg }} \rightarrow Q \mathcal{P}(M,-1)^{\text {nondeg }}
$$

is a weak equivalence. In particular, there is a morphism

$$
Q \mathcal{P}(M,-1)^{\text {nondeg }} \rightarrow G \operatorname{Sp}(A / R,-1)
$$

in the homotopy category of simplicial sets. 


\section{J. P. PRIDHAM}

Proof. We adapt the proof of [Pri17, Proposition 1.26]. For any $\Delta \in Q \mathcal{P}(M,-1)$, the homotopy fibre of $Q \operatorname{Comp}(A / R,-1)^{\text {nondeg }}$ over $\Delta$ is just the homotopy fibre of

$$
\mu(-, \Delta): G \operatorname{PreSp}(A / R,-1) \rightarrow T_{\Delta} Q^{\mathrm{tw}} \mathcal{P}(M,-1)
$$

over $-\partial_{\hbar^{-1}}(\Delta)$.

The map $\mu(-, \Delta): \operatorname{DR}^{\prime}(A / R) \llbracket \hbar \rrbracket \rightarrow T_{\Delta} Q \widehat{\operatorname{Pol}}(M,-1)$ is a morphism of complete $(G * \tilde{F})$ filtered $R \llbracket \hbar \rrbracket$-DGAAs by Lemma 1.33. Since the morphism is $R \llbracket \hbar \rrbracket$-linear, it maps $G^{k}(G *$ $\tilde{F})^{p} \operatorname{DR}^{\prime}(A / R) \llbracket \hbar \rrbracket$ to $G^{k}(G * \tilde{F})^{p} T_{\Delta} Q \widehat{\operatorname{Pol}}(M,-1)$. The non-degeneracy of $\Delta_{2}$ modulo $F_{1}$ implies that $\mu(-, \Delta)$ induces quasi-isomorphisms

$$
\Omega^{p-2 k} \hbar^{k}[2 k-p] \rightarrow \underline{\operatorname{Hom}}_{A}\left(\operatorname{CoS}^{p-2 k} \Omega_{A / R}^{1} \otimes_{A} M, M\right) \hbar^{p-k}
$$

on the associated graded pieces $\operatorname{gr}_{G}^{k} \operatorname{gr}_{(G * \tilde{F})}^{p}$. We therefore have a quasi-isomorphism of bifiltered complexes, so we have isomorphisms on homotopy groups:

$$
\begin{gathered}
\pi_{j} G \operatorname{PreSp}(A / R,-1) \rightarrow \pi_{j} T_{\Delta} Q^{\mathrm{tw}} \mathcal{P}(M,-1), \\
\mathrm{H}^{1-j}\left((G * \tilde{F})^{2} \operatorname{DR}(A / R) \llbracket \hbar \rrbracket\right) \rightarrow \mathrm{H}^{1-j}\left((G * \tilde{F})^{2} T_{\Delta} Q \widehat{\operatorname{Pol}}(M,-1)\right) .
\end{gathered}
$$

1.3. Comparing quantisations and generalised symplectic structures. We now investigate the extent to which the elements of a compatible pair determine each other.

Definition 1.39. Given a compatible pair $(\omega, \pi) \in \operatorname{Comp}(A,-1)=Q \operatorname{Comp}(M,-1) / G^{1}$ and $k \geqslant 0$, define the complex $N(\omega, \pi, k)$ to be the cocone of the map

$$
\operatorname{gr}_{G}^{k}(G * \tilde{F})^{2} \mathrm{DR}^{\prime}(A / R) \llbracket \hbar \rrbracket \oplus \operatorname{gr}_{G}^{k} \tilde{F}^{2} Q \widehat{\operatorname{Pol}}(M,-1) \rightarrow \operatorname{gr}_{G}^{k}(G * \tilde{F})^{2} T_{\pi} Q \widehat{\operatorname{Pol}}(M,-1)
$$

given by combining

$$
\begin{aligned}
& \operatorname{gr}_{G}^{k} \mu(-, \pi): \operatorname{gr}_{G}^{k}(G * \tilde{F})^{2} \mathrm{DR}^{\prime}(A / R) \llbracket \hbar \rrbracket \rightarrow \operatorname{gr}_{G}^{k}(G * \tilde{F})^{2} T_{\pi} Q \widehat{\operatorname{Pol}}(M,-1), \\
& F^{2-2 k} \operatorname{DR}(A / R) \hbar^{k} \rightarrow \prod_{i \geqslant(2-2 k), 0} \underline{\operatorname{Hom}}_{A}\left(\operatorname{CoS}_{A}^{i}\left(\Omega_{A / R}^{1}\right), A\right) \hbar^{i+k}
\end{aligned}
$$

with the maps

$$
\begin{gathered}
\operatorname{gr}_{G}^{k} \nu(\omega, \pi)+\partial_{\hbar^{-1}}:\left(\operatorname{gr}_{G}^{k} \tilde{F}^{2} Q \widehat{\operatorname{Pol}}(M,-1), \delta_{\pi}\right) \rightarrow \operatorname{gr}_{G}^{k}(G * \tilde{F})^{2} T_{\pi} Q \widehat{\operatorname{Pol}}(M,-1), \\
\prod_{i \geqslant(2-k), 0} \underline{\operatorname{Hom}}_{A}\left(\operatorname{CoS}_{A}^{i}\left(\Omega_{A / R}^{1}\right), A\right) \hbar^{i+k-1} \rightarrow \prod_{i \geqslant(2-2 k), 0} \underline{\operatorname{Hom}}_{A}\left(\operatorname{CoS}_{A}^{i}\left(\Omega_{A / R}^{1}\right), A\right) \hbar^{i+k},
\end{gathered}
$$

where $\nu(\omega, \pi)(b):=\nu(\omega, \pi, b)$.

It follows from the proof of Proposition 1.38 that the maps $\operatorname{gr}_{G}^{k} \mu(-, \pi)$ are all $F$-filtered quasiisomorphisms when $\pi$ is non-degenerate, so the projection maps $N(\omega, \pi, k) \rightarrow \operatorname{gr}_{G}^{k} \tilde{F}^{2} Q \widehat{\operatorname{Pol}}(M,-1)$ are also quasi-isomorphisms. The behaviour of the other projection is more subtle for low $k$, but it behaves well thereafter.

Lemma 1.40. The projection maps $N(\omega, \pi, k) \rightarrow \hbar^{k} \mathrm{DR}(A / R)$ are $F$-filtered quasi-isomorphisms for all $k \geqslant 2$.

Proof. This amounts to showing that the map $\operatorname{gr}_{G}^{k} \nu(\omega, \pi)+\partial_{\hbar^{-1}}$ is a filtered quasi-isomorphism. It suffices to show that the associated maps

$$
\begin{gathered}
\operatorname{gr}_{F}^{p} \operatorname{gr}_{G}^{k} \nu(\omega, \pi)+\partial_{\hbar^{-1}}: \operatorname{gr}_{F}^{p} \operatorname{gr}_{G}^{k}(G * \tilde{F})^{2} Q \widehat{\operatorname{Pol}}(M,-1) \rightarrow \operatorname{gr}_{F}^{p} \operatorname{gr}_{G}^{k}(G * \tilde{F})^{2} T_{\pi} Q \widehat{\operatorname{Pol}}(M,-1), \\
\underline{\operatorname{Hom}}_{A}\left(\operatorname{CoS}_{A}^{p}\left(\Omega_{A / R}^{1}\right), A\right) \hbar^{p+k-1} \rightarrow \underline{\operatorname{Hom}}_{A}\left(\operatorname{CoS}_{A}^{p}\left(\Omega_{A / R}^{1}\right), A\right) \hbar^{p+k}
\end{gathered}
$$




\section{QUANTISING (-1)-SHIFTED SYMPLECTIC STRUCTURES}

are quasi-isomorphisms for all $p \geqslant 0$.

Reasoning as in [Pri17, Lemma 1.34], we have that $\operatorname{gr}_{F} \operatorname{gr}_{G} \nu(\omega, \pi)$ is an $R \llbracket \hbar \rrbracket$-linear derivation on $\operatorname{gr}_{F} \operatorname{gr}_{G} Q \widehat{\operatorname{Pol}}(M,-1) \cong \widehat{\operatorname{Pol}}(A,-1) \llbracket \hbar \rrbracket$ with respect to the commutative multiplication. It is given on generators $\underline{\operatorname{Hom}}_{A}\left(\Omega_{A / R}^{1}, A\right)$ by $\hbar \pi^{\sharp} \circ \omega^{\sharp}$. The compatibility of $\omega$ and $\pi$ implies that $\pi^{\sharp} \circ \omega^{\sharp}$ is homotopy idempotent by [Pri17, Example 1.19]. Thus $\hbar^{-1} \operatorname{gr}_{F}^{p} \operatorname{gr}_{G}^{k} \nu(\omega, \pi)$ is homotopy diagonalisable, with integral eigenvalues in the interval $[0, p]$.

On the other hand, $\partial_{\hbar^{-1}}$ coincides on $\operatorname{gr}_{F}^{p} \operatorname{gr}_{G}^{k}$ with multiplication by $(1-p-k) \hbar$, so the eigenvalues of $\hbar^{-1} \operatorname{gr}_{F}^{p} \operatorname{gr}_{G}^{k} \nu(\omega, \pi)+\hbar^{-1} \partial_{\hbar^{-1}}$ lie in $[1-p-k, 1-k]$, giving a quasi-isomorphism when $k>1$.

Proposition 1.41. The maps

$$
\begin{aligned}
Q \mathcal{P}(M,-1)^{\text {nondeg }} / G^{k} & \rightarrow\left(Q \mathcal{P}(M,-1)^{\text {nondeg }} / G^{2}\right) \times{ }_{\left(G \operatorname{Sp}(A,-1) / G^{2}\right)}^{h}\left(G \operatorname{Sp}(A,-1) / G^{k}\right) \\
& \simeq\left(Q \mathcal{P}(M,-1)^{\text {nondeg }} / G^{2}\right) \times \prod_{i=2}^{k-1} \underline{\operatorname{MC}}\left(\operatorname{DR}(A / R) \hbar^{i}\right)
\end{aligned}
$$

coming from Proposition 1.38 are weak equivalences for all $k \geqslant 2$.

Proof. Proposition 1.38 gives equivalences between $Q \mathcal{P}^{\text {nondeg }}$ and $Q$ Comp ${ }^{\text {nondeg }}$. Fix $(\omega, \pi) \in$ $\operatorname{Comp}(A,-1)$, and denote homotopy fibres by subscripts. Arguing as in the proof of [Pri17, Proposition 1.35], but with abelian (rather than central) extensions of DGLAs as in [Pri13a, Lemma 3.3], gives a commutative diagram

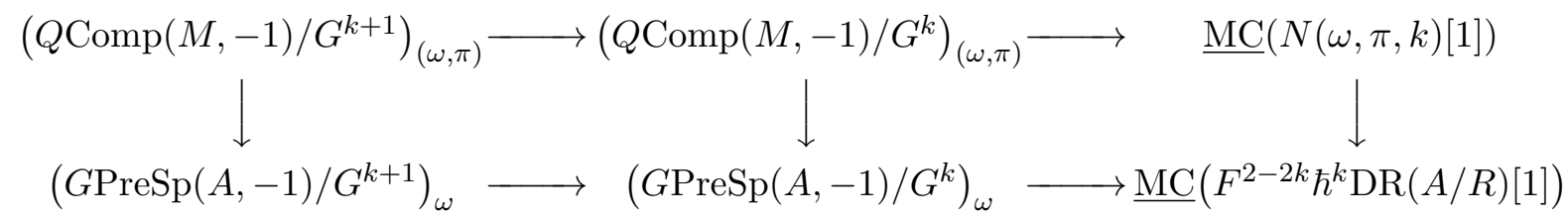

of fibre sequences.

The right-hand map is a weak equivalence for $k \geqslant 2$, by $\operatorname{Lemma} 1.40$, so $Q \operatorname{Comp}(M,-1) / G^{k+1}$ is equivalent to the homotopy fibre product

$$
\left(Q \operatorname{Comp}(M,-1) / G^{k}\right) \times{ }_{G \operatorname{PreSp}(A,-1) / G^{k}}^{h} G \operatorname{PreSp}(A,-1) / G^{k+1},
$$

and the result follows by induction.

Remark 1.42. Taking the limit over all $k$, we see that Proposition 1.41 gives an equivalence

$$
Q \mathcal{P}(M,-1)^{\text {nondeg }} \simeq\left(Q \mathcal{P}(M,-1)^{\text {nondeg }} / G^{2}\right) \times \prod_{i \geqslant 2} \underline{\mathrm{MC}}\left(\mathrm{DR}(A / R) \hbar^{i}\right) ;
$$

in particular, this means that there is a canonical map

$$
\left(Q \mathcal{P}(M,-1)^{\text {nondeg }} / G^{2}\right) \rightarrow Q \mathcal{P}(M,-1)^{\text {nondeg }}
$$

corresponding to the distinguished point $0 \in \underline{\mathrm{MC}}\left(\hbar^{2} \mathrm{DR}(A / R) \llbracket \hbar \rrbracket\right)$.

Thus to quantise a non-degenerate $(-1)$-shifted Poisson structure $\pi=\sum_{j \geqslant 2} \pi_{j}$ (or equivalently, by [Pri17, Corollary 1.38], a (-1)-shifted symplectic structure), it suffices to lift the power series $\sum_{j \geqslant 2} \pi_{j}(-\hbar)^{j-1}$ to a Maurer-Cartan element of $\prod_{j \geqslant 2}\left(F_{j} \mathscr{D}(M) / F_{j-2}\right) \hbar^{j-1}$.

Even in the degenerate case, the proof of Proposition 1.41 gives a sufficient first-order criterion for quantisations to exist:

$$
Q \operatorname{Comp}(M,-1) \simeq\left(Q \operatorname{Comp}(M,-1) / G^{2}\right) \times \prod_{i \geqslant 2} \underline{\mathrm{MC}}\left(\mathrm{DR}(A / R) \hbar^{i}\right) .
$$




\section{J. P. PRIDHAM}

\section{Quantisation for derived DM N-stacks}

In this section, we will globalise the results of the previous section to the setting of derived DM $N$-stacks. In order to pass from derived affine schemes to derived DM stacks, we will exploit étale functoriality using Segal spaces.

The basic idea is that given a small category $I$, an $I$-diagram $A$ of CDGAs, and an $A$ module $M$ in $I$-diagrams, we can construct a DGAA $\mathscr{D}_{A}(M)$ of differential operators of $M$. When $M$ is a strict line bundle, $\mathscr{D}_{A}(M)$ then gives rise to a filtered DGLA $Q \widehat{\operatorname{Pol}}(M,-1)$ governing $E_{0}$ quantisations of the diagram $M$.

If we could choose appropriate restrictions on $(A, M)$ to ensure that $\mathscr{D}_{A}(M)$ had the correct homotopical properties, passage to Maurer-Cartan spaces would then naturally give a presentation of the $\infty$-category of $E_{0}$ quantisations as a derivator. However, this is not straightforward, since in order to compute both de Rham and Poisson cohomology correctly, we need the $A$ modules $\Omega_{A}^{m}$ to be both fibrant and cofibrant.

When $I$ is a category of the form $[m]=(0 \rightarrow 1 \rightarrow \cdots \rightarrow m)$ and $A$ is fibrant and cofibrant in the injective model structure, this condition is satisfied, so we can construct Maurer-Cartan spaces of $[m]$-diagrams, providing all the data necessary to form Segal spaces.

2.1. Quantised polyvectors for diagrams. We now construct differential operators and quantised polyvectors for suitable diagrams of derived affine schemes.

Definition 2.1. Given a small category $I$, an $I$-diagram $A$ of $R$-CDGAs, and $A$-modules $M$ and $N$ in $I$-diagrams of cochain complexes, define the filtered cochain complex $\operatorname{Diff}(M, N)=$ $\operatorname{Diff}_{A / R}(M, N) \subset \underline{\operatorname{Hom}}_{R}(M, N)$ of differential operators from $M$ to $N$ as the equaliser of the obvious diagram

$$
\prod_{i \in I} \operatorname{Diff}_{A(i) / R}(M(i), N(i)) \Longrightarrow \prod_{f: i \rightarrow j \text { in } I} \operatorname{Diff}_{A(i) / R}\left(M(i), f_{*} N(j)\right)
$$

with the filtration $F_{k} \operatorname{Diff}(M, N)$ defined similarly.

We then write $\mathscr{D}(M)=\mathscr{D}_{A / R}(M):=\operatorname{Diff}_{A / R}(M, M)$, which we regard as a DGAA under composition. We simply write $\mathscr{D}_{A}=\mathscr{D}_{A / R}$ for $\mathscr{D}_{A / R}(A, A)$.

For $f: i \rightarrow j$ a morphism in $I$, the maps

$$
\operatorname{gr}_{k}^{F} \operatorname{Diff}_{A(i) / R}\left(M(i), f_{*} N(j)\right) \rightarrow \underline{\operatorname{Hom}}_{A(i)}\left(M(i) \otimes_{A(i)} \operatorname{CoS}_{A(i) / R}^{k} \Omega_{A(i)}^{1}, f_{*} N(j)\right)
$$

are isomorphisms whenever $A(i)$ is semi-smooth and $M(i)^{\sharp}$ projective over $A(i)^{\sharp}$. When these conditions hold for all $i$, the maps

$$
\operatorname{gr}_{k}^{F} \operatorname{Diff}_{A / R}(M, N) \rightarrow \underline{\operatorname{Hom}}_{A}\left(M \otimes_{A} \operatorname{CoS}_{A}^{k} \Omega_{A / R}^{1}, N\right)
$$

are thus also isomorphisms.

We now have analogues of all the constructions in Sections 1.1.3 and 1.1.4.

Definition 2.2. Given an $I$-diagram $A$ of $R$-CDGAs and an $I$-diagram $M$ of strict line bundles 


\section{QUANTISING (-1)-SHIFTED SYMPLECTIC STRUCTURES}

over $A$, define the filtered DGLA of quantised (-1)-shifted polyvector fields on $M$ by

$$
\begin{aligned}
Q \widehat{\operatorname{Pol}}(M,-1) & :=\prod_{j \geqslant 0} F_{j} \mathscr{D}_{A / R}(M) \hbar^{j-1}, \\
\tilde{F}^{i} Q \widehat{\operatorname{Pol}}(M,-1) & :=\prod_{j \geqslant i} F_{j} \mathscr{D}_{A / R}(M) \hbar^{j-1}, \\
G^{k} Q \widehat{\operatorname{Pol}}(M,-1) & :=\hbar^{k} Q \widehat{\operatorname{Pol}}(M,-1) .
\end{aligned}
$$

Definition 2.3. We then define $Q \mathcal{P}(M,-1), T Q \widehat{\operatorname{Pol}}(M,-1), T_{\Delta} Q \widehat{\operatorname{Pol}}(M,-1), T Q \mathcal{P}(M,-1)$, $T_{\Delta} Q \mathcal{P}(M,-1), \sigma=-\partial_{\hbar^{-1}}: Q \widehat{\operatorname{Pol}}(M,-1) \rightarrow T Q \widehat{\operatorname{Pol}}(M,-1)$, and $\sigma: Q \mathcal{P}(M,-1) \rightarrow T Q \mathcal{P}(M,-1)$ as before, replacing Definition 1.10 with Definition 2.2.

Note that if $u: I \rightarrow J$ is a morphism of small categories and $A$ is a functor from $J$ to $R$ CDGAs with $B=A \circ u$, then we have natural maps $F(A) \rightarrow F(B)$ for all the constructions $F$ of Definition 2.3.

Lemma 2.4 ([Pri17, Lemma 2.2]). If $A$ is an $[n]$-diagram in $R$-CDGAs which is cofibrant and fibrant for the injective model structure (that is, each $A(i)$ is cofibrant and the maps $A(i) \rightarrow$ $A(i+1)$ are surjective), then $\underline{\operatorname{Hom}}_{A}\left(\operatorname{CoS}_{A}^{k} \Omega_{A}^{1}, A\right)$ is a model for the derived Hom-complex $\mathbf{R} \underline{\operatorname{Hom}}_{A}\left(\mathbf{L C o S}{ }_{A}^{k} \mathbf{L} \Omega_{A}^{1}, A\right)$, and $\underline{\operatorname{Hom}}_{A}\left(A, \Omega_{A}^{m}\right) \simeq \operatorname{holim}_{i} \mathbf{L} \Omega_{A(i)}^{m}$.

When $A$ satisfies the conditions of Lemma 2.4, the lemma combines with the observation above to show that every strict line bundle $M$ over $A$ satisfies

$$
\operatorname{gr}_{k}^{F} \mathscr{D}_{A / R}(M) \simeq \mathbf{R} \underline{\operatorname{Hom}}_{A}\left(\mathbf{L C o S}{ }_{A}^{k} \mathbf{L} \Omega_{A / R}^{1}, A\right) .
$$

Definition 2.5. Given an $[m]$-diagram $A$ satisfying the conditions of Lemma 2.4, define

$$
G \operatorname{PreSp}(A / R,-1):=G \operatorname{PreSp}(A(0) / R,-1)=\lim _{i \in[m]} G \operatorname{PreSp}(A(i) / R,-1)
$$

for the space GPreSp of generalised pre-symplectic structures of Definition 1.30.

For a strict line bundle $M$ over $A$, define

$$
\mu: G \operatorname{PreSp}(A / R,-1) \times Q \mathcal{P}(M,-1) \rightarrow T Q^{\mathrm{tw}} \mathcal{P}(M,-1)
$$

by setting $\mu(\omega, \Delta)(i):=\mu(\omega(i), \Delta(i)) \in T Q \mathcal{P}(M(i),-1)$ for $i \in[m]$, and let $Q \operatorname{Comp}(M,-1)$ be the homotopy vanishing locus of

$$
(\mu-\sigma): G \operatorname{PreSp}(A / R,-1) \times Q \mathcal{P}(M,-1) \rightarrow T Q^{\mathrm{tw}} \mathcal{P}(M,-1) .
$$

over $Q^{\mathrm{tw}} \mathcal{P}(M,-1)$.

Lemma 2.6 ([Pri17, Lemma 2.6]). If $D=(A \rightarrow B)$ is a fibrant cofibrant [1]-diagram of $R$-CDGAs which is formally étale in the sense that the map $\Omega_{A}^{1} \otimes_{A} B \rightarrow \Omega_{B}^{1}$ is a quasi-isomorphism, then the map

$$
\underline{\operatorname{Hom}}_{D}\left(\operatorname{CoS}_{D}^{k} \Omega_{D}^{1}, D\right) \rightarrow \underline{\operatorname{Hom}}_{A}\left(\operatorname{CoS}_{A}^{k} \Omega_{A}^{1}, A\right)
$$

is a quasi-isomorphism.

2.2. Towers of obstructions. We now show how to adapt the various obstruction towers from Section 1 to apply to $[m]$-diagrams of derived affines. 


\section{J. P. PRIDHAM}

Definition 2.7. For an $[m]$-diagram $A$ and $k \geqslant 1$, define

$$
\begin{aligned}
& \mathrm{Ob}(Q \mathcal{P}, A, k):=\underline{\mathrm{MC}}\left(F^{2} \widehat{\operatorname{Pol}}(A,-1) \oplus \hbar^{k} F^{2-k} \widehat{\operatorname{Pol}}(A,-1)[1]\right), \\
& \mathrm{Ob}\left(Q^{\mathrm{tw}} \mathcal{P}, A, k\right):=\underline{\mathrm{MC}}\left(F^{2} \widehat{\operatorname{Pol}}(A,-1) \oplus \hbar^{k} F^{2-2 k} \widehat{\operatorname{Pol}}(A,-1)[1]\right),
\end{aligned}
$$

where the DGLA structure is defined by regarding the second term as a module over the first. Note that these expressions only differ for $k=1$, as $F^{<0}=F^{0}$.

Projection gives a fibration $\operatorname{Ob}(Q \mathcal{P}, A, k) \rightarrow \mathcal{P}(A,-1)=Q \mathcal{P}(A,-1) / G^{1}$, with the fibre over $\pi$ being $\underline{\mathrm{MC}}\left(\hbar^{k} F^{2-k} T_{\pi} \widehat{\mathrm{Pol}}(A,-1)[1]\right)$, and similarly for $\mathrm{Ob}\left(Q^{\mathrm{tw}} \mathcal{P}, A, k\right)$.

For strict line bundles $M$ over $I$-diagrams $A$, the extension

$$
\tilde{F}^{2} Q \widehat{\operatorname{Pol}}(M,-1) / G^{k+1} \rightarrow \tilde{F}^{2} Q \widehat{\operatorname{Pol}}(M,-1) / G^{k}
$$

of DGLAs, with abelian kernel $\hbar^{k} F^{2-k} \widehat{\operatorname{Pol}}(A,-1)$, and its analogue for $(G * \tilde{F})^{2}$ give rise to the following canonical fibration sequences over $\mathcal{P}(A,-1)$ :

$$
\begin{gathered}
Q \mathcal{P}(M,-1) / G^{k+1} \rightarrow Q \mathcal{P}(M,-1) / G^{k} \stackrel{\mathrm{ob}}{\longrightarrow} \mathrm{Ob}(Q \mathcal{P}, A, k), \\
Q^{\mathrm{tw}} \mathcal{P}(M,-1) / G^{k+1} \rightarrow Q^{\mathrm{tw}} \mathcal{P}(M,-1) / G^{k} \stackrel{\mathrm{ob}}{\longrightarrow} \mathrm{Ob}\left(Q^{\mathrm{tw}} \mathcal{P}, A, k\right) .
\end{gathered}
$$

Similarly, we have a fibration sequence

$$
G \operatorname{PreSp}(A / R,-1) / G^{k+1} \rightarrow G \operatorname{PreSp}(A / R,-1) / G^{k} \stackrel{\text { ob }}{\longrightarrow} \underline{\operatorname{MC}}\left(\hbar^{k} \operatorname{DR}(A(0) / R)[1]\right) .
$$

We also have a map $\sigma=-\partial_{\hbar^{-1}}: \operatorname{Ob}(Q \mathcal{P}, A, k) \rightarrow \hbar \mathrm{Ob}(Q \mathcal{P}, A, k)$ and maps

$$
\begin{gathered}
\operatorname{PreSp}(A / R,-1) \times \mathrm{Ob}(Q \mathcal{P}, A, k) \stackrel{\nu}{\rightarrow} \hbar \mathrm{Ob}\left(Q^{\mathrm{tw}} \mathcal{P}, A, k\right), \\
\underline{\mathrm{MC}}\left(\hbar^{k} \operatorname{DR}(A(0) / R)[1]\right) \times \mathcal{P}(A,-1) \stackrel{\mu}{\rightarrow} \hbar \mathrm{Ob}\left(Q^{\mathrm{tw}} \mathcal{P}, A, k\right)
\end{gathered}
$$

given by $\nu(\omega, \pi, u)(i)=(\pi(i), \nu(\omega(i), \pi(i), u(i)))$ and $\mu(v, \pi)(i)=(\pi(i), \mu(v(i), \pi(i)))$ for $i \in[m]$.

Definition 2.8. For $k \geqslant 1$, define $\operatorname{Ob}(Q \operatorname{Comp} / Q \mathcal{P}, A, k)$ to be the homotopy vanishing locus of

$$
\mu: \underline{\mathrm{MC}}\left(\hbar^{k} \mathrm{DR}(A(0) / R)[1]\right) \times \mathcal{P}(A,-1) \rightarrow \hbar \mathrm{Ob}\left(Q^{\mathrm{tw}} \mathcal{P}, A, k\right)
$$

over $\mathcal{P}(A,-1)$

Combining the earlier fibration sequences with the definition of $Q$ Comp, we have the following.

Lemma 2.9. There is a natural obstruction map

ob: $\left(Q \operatorname{Comp}(M,-1) / G^{k}\right) \times_{\left(Q \mathcal{P}(M,-1) / G^{k}\right)}^{h}\left(Q \mathcal{P}(M,-1) / G^{k+1}\right) \rightarrow \mathrm{Ob}(Q \operatorname{Comp} / Q \mathcal{P}, A, k)$

over $\mathcal{P}(A,-1)$, whose homotopy vanishing locus is $Q \operatorname{Comp}(M,-1) / G^{k+1}$.

Definition 2.10. Define $\mathrm{Ob}(Q \operatorname{Comp} / Q \mathcal{S}, A, k)$ to be the homotopy vanishing locus of

$$
\left(\nu-\sigma \circ \operatorname{pr}_{2}\right): \operatorname{Comp}(A,-1) \times_{\mathcal{P}(A,-1)} \mathrm{Ob}(Q \mathcal{P}, A, k) \rightarrow \hbar \mathrm{Ob}\left(Q^{\mathrm{tw}} \mathcal{P}, A, k\right)
$$

over $\mathcal{P}(A,-1)$.

Combining the earlier fibration sequences with the definition of $Q$ Comp, we also have the following.

LEMMA 2.11. There is a natural obstruction map

ob: $\left(Q \operatorname{Comp}(M,-1) / G^{k}\right) \times_{\left(G \operatorname{PreSp}(A,-1) / G^{k}\right)}^{h}\left(G \operatorname{PreSp}(A,-1) / G^{k+1}\right) \rightarrow \operatorname{Ob}(Q \operatorname{Comp} / Q \mathcal{S}, A, k)$

over $\operatorname{Comp}(A,-1)$, whose homotopy vanishing locus is $Q \operatorname{Comp}(M,-1) / G^{k+1}$. 


\section{QUANTISING (-1)-SHIFTED SYMPLECTIC STRUCTURES}

2.3. Descent and line bundles. In order to define our various structures on derived DM $N$-stacks and to look at quantisations of line bundles, we now make use of étale descent and functoriality.

Definition 2.12. Write $d g \operatorname{CAlg}(R)$ for the category of CDGAs over $R$, and let $d g \mathrm{CAlg}(R)_{c, \rightarrow} \subset$ $d g \mathrm{CAlg}(R)$ be the subcategory with all cofibrant $R$-CDGAs as objects, and only surjective morphisms.

We already have simplicial set-valued functors $G \operatorname{PreSp}(-,-1)$ and $G \operatorname{Sp}(-,-1)$ from $d g \mathrm{CAlg}(R)$ to $s$ Set, mapping quasi-isomorphisms in $d g \mathrm{CAlg}(R)_{c}$ to weak equivalences. Poisson structures and their quantisations are only functorial with respect to formally étale morphisms, in an $\infty$-functorial sense which we now make precise.

Observe that when $F$ is any of the constructions $Q \mathcal{P}(-,-1), Q \operatorname{Comp}(-,-1), G \operatorname{PreSp}(-,-1)$, $\mathrm{Ob}(Q \mathcal{P},-, k), \mathrm{Ob}(Q \operatorname{Comp} / Q \mathcal{P},-, k)$, or $\mathrm{Ob}(Q \operatorname{Comp} / Q \mathcal{S},-, k)$ applied to $[m]$-diagrams in $d g \operatorname{CAlg}(R)_{c \rightarrow \rightarrow}$, Lemmas 2.4 and 2.6 combine with the obstruction calculus of Section 2.2 to show that the following are satisfied.

Properties 2.13 ([Pri17, Properties 2.5]). (1) The maps from $F(A(0) \rightarrow \cdots \rightarrow A(m))$ to

$$
F(A(0) \rightarrow A(1)) \times_{F(A(1)}^{h} F(A(1) \rightarrow A(2)) \times_{F(A(2)}^{h} \cdots \times_{F(A(n-1)}^{h} F(A(n-1) \rightarrow A(n))
$$

are weak equivalences.

(2) If the [1]-diagram $A \rightarrow B$ is a quasi-isomorphism, then the natural maps from $F(A \rightarrow B)$ to $F(A)$ and to $F(B)$ are weak equivalences.

(3) If the [1]-diagram $A \rightarrow B$ is formally étale, then the natural map from $F(A \rightarrow B)$ to $F(A)$ is a weak equivalence.

The first two properties ensure that the simplicial classes $\coprod_{A \in B_{m} d g \operatorname{CAlg}(R)_{c \rightarrow \rightarrow}} F(A)$ fit together to give a complete Segal space $\int F$ over the nerve $B d g \operatorname{CAlg}(R)_{c \rightarrow \rightarrow}$. Taking Segal spaces as our preferred model of $\infty$-categories, we define $\mathbf{L} d g \mathrm{CAlg}(R)_{c \rightarrow \rightarrow}$ and $\mathbf{L} d g \mathrm{CAlg}(R)$ to be the $\infty$ categories obtained by localising the respective $(\infty$-)categories at quasi-isomorphisms or weak equivalences, and let $\mathbf{L} d g \mathrm{CAlg}(R)^{\text {ét }} \subset \mathbf{L} d g \mathrm{CAlg}(R)$ be the $\infty$-subcategory of homotopy formally étale morphisms.

For any construction $F$ satisfying the conditions above, [Pri17, Definition 2.7] then gives an $\infty$-functor

$$
\mathbf{R} F: \mathbf{L} d g \mathrm{CAlg}(R)^{\mathrm{e} t} \rightarrow \mathbf{L} s \operatorname{Set}
$$

to the $\infty$-category of simplicial sets, with the property that $(\mathbf{R} F)(A) \simeq F(A)$ for all cofibrant $R$-CDGAs $A$.

Definition 2.14. Given a derived Deligne-Mumford $N$-stack $\mathfrak{X}$ and any of the constructions $F$ above, define $F(\mathfrak{X})$ to be the homotopy limit of $\mathbf{R} F(A)$ over the $\infty$-category ( $D G$ Affét $\downarrow \mathfrak{X}$ ) consisting of derived affines $\operatorname{Spec} A$ equipped with homotopy étale (that is, étale in the sense of [TV08]) maps to $\mathfrak{X}$ and all homotopy étale morphisms between them.

When $\mathfrak{X} \simeq \operatorname{Spec} B$ is a derived affine, note that it is final in the category of derived affines over $\mathfrak{X}$, so $\mathbf{R} F(\mathfrak{X})=\mathbf{R} F(B)=F(B)$. In general, it suffices to take the homotopy limit over any subcategory of ( $D G$ Affét $\downarrow \mathfrak{X})$ with colimit $\mathfrak{X}$, so this definition also coincides with [Pri17, Definition 2.18], by applying it to a suitable hypergroupoid. 


\section{J. P. PRIDHAM}

Definition 2.14 is insufficient for our purposes, as we wish to consider line bundles. Since DM stacks only involve CDGAs with non-positive cohomology, the line bundles we encounter will be locally trivial, so for now we only need to set up $\mathbb{G}_{m}$-equivariance.

Definition 2.15. Define the functor $\mathbb{G}_{m}$ from CDGAs to groups by

$$
\mathbb{G}_{m}(A):=\mathrm{Z}^{0}(A)^{\times} \text {. }
$$

Given a string $A=(A(0) \rightarrow \cdots \rightarrow A(n))$ of CDGAs, we write $\mathbb{G}_{m}(A):=\mathbb{G}_{m}(A(0))$, regarded as $\lim _{\leftarrow} \mathbb{G}_{m}(A(i))$.

Now, the group $\mathbb{G}_{m}(A)^{\times}$acts by conjugation on $Q \widehat{\operatorname{Pol}}(A,-1)$, corresponding to automorphisms of $A$ as a line bundle over $A$. This action preserves all the filtrations, so it acts on the simplicial set $Q \mathcal{P}(A,-1)=\underline{\mathrm{MC}}\left(\tilde{F}^{2} Q \widehat{\operatorname{Pol}}(A,-1)\right)$ respecting the cofiltrations. Note that the action is trivial on the quotient $Q \mathcal{P}(A,-1) / G^{1}=\mathcal{P}(A,-1)$.

Definition 2.16. For any of the constructions $F$ above, let $\mathbf{R}\left(F /{ }^{h} \mathbb{G}_{m}\right)$ be the $\infty$-functor on $\mathbf{L} d g \mathrm{CAlg}(R)^{\text {ét }}$ given by applying the construction of [Pri17, Definition 2.18] to the homotopy quotient $F /{ }^{h} \mathbb{G}_{m}$, then taking étale hypersheafification.

Up to now, hypersheafification has not been necessary because all our functors have been hypersheaves - this follows because the associated graded pieces $\operatorname{gr}_{F}$ of obstruction functors can be written in terms of tangent sheaves and sheaves of differential forms. However, $B \mathbb{G}_{m}$ requires hypersheafification because the simplicial presheaf $B \mathbb{G}_{m}$ does not preserve weak equivalences or satisfy étale descent.

For any derived line bundle $\mathscr{L}$ on a derived stack $\mathfrak{X}$, there is an associated $\mathbb{G}_{m}$-torsor given locally by the disjoint union of spaces of quasi-isomorphisms from $\mathscr{O}_{\mathfrak{X}}[m]$ to $\mathscr{L}$ for all $m \in \mathbb{Z}$.

Definition 2.17. Given a derived Deligne-Mumford $N$-stack $\mathfrak{X}$, a derived line bundle $\mathscr{L}$ on $\mathfrak{X}$, and any of the constructions $F$ above, define $F(\mathscr{L})$ to be the homotopy limit of $\mathbf{R}\left(F /{ }^{h} \mathbb{G}_{m}\right)(A)$

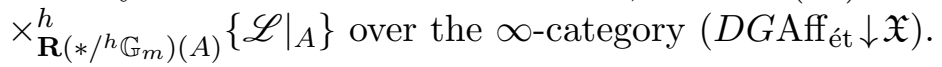

Remarks 2.18. If we fix $\pi \in \mathcal{P}(\mathfrak{X},-1)$, then observe that the homotopy fibres of $Q \mathcal{P}(\mathscr{L},-1) \rightarrow$ $Q \mathcal{P}(\mathscr{L},-1) / G^{1}=\mathcal{P}(M,-1)$ over $\pi$ can be combined and enhanced to a dg category whose objects are $E_{0}$ quantisations $(\mathscr{L}, \Delta)$ over $\pi$, with $\mathrm{dg}$ morphisms $(\mathscr{L}, \Delta) \rightarrow\left(\mathscr{L}^{\prime}, \Delta^{\prime}\right)$ given by the complex $\left(\prod_{j \geqslant 0} F_{j} \mathscr{D i f f}_{\mathfrak{X} / R}\left(\mathscr{L}, \mathscr{L}^{\prime}\right) \hbar^{j},\left(\delta+\Delta^{\prime}\right)_{*} \mp f(\delta+\Delta)^{*}\right)$ and the obvious composition law.

Also note that the action of $\mathbb{G}_{m}(A)^{\times}$is unipotent, as it is trivial on $\mathcal{P}(A,-1)$, so it extends naturally to an action of $\mathbb{G}_{m}(A)^{\times} \otimes_{\mathbb{Z}} \mathbb{Q}$, and we can therefore define quantisations for étale $B\left(\mathbb{G}_{m} / \mu_{\infty}\right)$-torsors. In fact, more is true: line bundles $\mathscr{M}$ with left $\mathscr{D}$-module structure give equivalences $\mathscr{D}(\mathscr{L}) \simeq \mathscr{D}(\mathscr{L} \otimes \mathscr{M})$ and hence $Q \mathcal{P}(\mathscr{L},-1) \simeq Q \mathcal{P}(\mathscr{L} \otimes \mathscr{M},-1)$. By sheafifying these equivalences, we get a notion of quantisation for all elements of $\underline{\mathrm{MC}}\left(F^{1} \mathrm{DR}(\mathfrak{X} / R)[1]\right)$ via Chern classes.

2.4. Comparing quantisations and generalised symplectic structures. We now fix a strongly quasi-compact derived DM $N$-stack $\mathfrak{X}$ over $R$.

Lemma 2.19. For $(\omega, \pi) \in \operatorname{Comp}(\mathfrak{X},-1)$, the homotopy fibre $\operatorname{Ob}(Q \operatorname{Comp} / Q \mathcal{S}, \mathfrak{X}, k)_{(\omega, \pi)}$ of $\operatorname{Ob}(Q \operatorname{Comp} / Q \mathcal{S}, \mathfrak{X}, k) \rightarrow \operatorname{Comp}(\mathfrak{X},-1)$ over $(\omega, \pi)$ is contractible for all $k \geqslant 2$.

Proof. As in the proof of Lemma 1.40, the map $\hbar^{-1} \nu(\omega, \pi,-)-(p-1)$ on

$$
\hbar^{p-1} \operatorname{Ext}_{\mathscr{O}_{\mathfrak{X}}}^{2-i}\left(\mathbf{L C o S}_{\mathscr{O}_{\mathfrak{X}}}^{p-k} \mathbf{L} \Omega_{\mathfrak{X} / R}^{1}, \mathscr{O}_{\mathfrak{X}}\right)
$$




\section{QuANTISING (-1)-SHIFTED SYMPLECTIC STRUCTURES}

is invertible for $k \geqslant 2$, so Lemma 2.11 gives contractibility of the homotopy fibre.

Proposition 2.20. For any line bundle $\mathscr{L}$ on $\mathfrak{X}$, the map

$$
\begin{aligned}
Q \operatorname{Comp}(\mathscr{L},-1) & \rightarrow\left(Q \operatorname{Comp}(\mathscr{L},-1) / G^{2}\right) \times{ }_{\left(G \operatorname{PreSp}(\mathfrak{X},-1) / G^{2}\right)}^{h} G \operatorname{PreSp}(\mathfrak{X},-1) \\
& \simeq\left(Q \operatorname{Comp}(\mathscr{L},-1) / G^{2}\right) \times \prod_{i \geqslant 2} \underline{\operatorname{MC}}\left(\operatorname{DR}(\mathfrak{X} / R) \hbar^{i}\right)
\end{aligned}
$$

is a weak equivalence.

Proof. This is essentially the same as the proof of Proposition 1.41. Lemma 2.19 combines with the obstruction maps

$$
\left(Q \operatorname{Comp}(\mathscr{L},-1) / G^{k}\right) \times_{\left(G \operatorname{PreSp}(\mathfrak{X},-1) / G^{k}\right)}^{h}\left(G \operatorname{PreSp}(\mathfrak{X},-1) / G^{k+1}\right) \rightarrow \operatorname{Ob}(Q \operatorname{Comp} / Q \mathcal{S}, \mathfrak{X}, k)
$$

to give the weak equivalences

$\left(Q \operatorname{Comp}(\mathscr{L},-1) / G^{k+1}\right) \simeq\left(Q \operatorname{Comp}(\mathscr{L},-1) / G^{k}\right) \times_{\left(G \operatorname{PreSp}(\mathfrak{X},-1) / G^{k}\right)}^{h}\left(G \operatorname{PreSp}(\mathfrak{X},-1) / G^{k+1}\right)$.

Definition 2.21. Given a (-1)-shifted Poisson structure $\pi \in \mathcal{P}(\mathfrak{X},-1)$, we say that $\pi$ is nondegenerate if the induced map

$$
\pi^{\sharp}: \mathbf{L} \Omega_{\mathfrak{X}}^{1} \rightarrow \mathbf{R} \mathcal{H}_{\operatorname{om}_{\mathscr{O}_{X}}}\left(\mathbf{L} \Omega_{\mathfrak{X}}^{1}, \mathscr{O}_{\mathfrak{X}}\right)[1]
$$

is a quasi-isomorphism of sheaves on $\mathfrak{X}$ and $\mathbf{L} \Omega_{\mathfrak{X}}^{1}$ is perfect.

Lemma 2.22. If $\pi$ is a non-degenerate $(-1)$-shifted Poisson structure on $\mathfrak{X}$, then the homotopy fibre $\mathrm{Ob}(Q \operatorname{Comp} / Q \mathcal{P}, \mathfrak{X}, k)_{\pi}$ of $\mathrm{Ob}(Q \operatorname{Comp} / Q \mathcal{P}, \mathfrak{X}, k) \rightarrow \mathcal{P}(\mathfrak{X},-1)$ over $\pi$ is contractible.

Proof. The map

$$
\mu(-, \pi): \mathrm{H}^{2-i}\left(\mathfrak{X}, \mathbf{L} \Omega_{\mathfrak{X} / R}^{p-2 k}\right) \rightarrow \hbar^{p} \operatorname{Ext}_{\mathscr{O}_{\mathfrak{X}}}^{2-i}\left(\mathbf{L C o S}_{\mathscr{O}_{\mathfrak{X}}}^{p-2 k} \mathbf{L} \Omega_{\mathfrak{X} / R}^{1}, \mathscr{O}_{\mathfrak{X}}\right)
$$

is given by $\Lambda^{p-2 k} \pi^{\sharp}$, so is an isomorphism by the non-degeneracy of $\pi$. Lemma 2.9 then gives the contractibility of $\mathrm{Ob}(Q \mathrm{Comp} / Q \mathcal{P}, \mathfrak{X}, k)_{\pi}$.

Proposition 2.23. For any line bundle $\mathscr{L}$ on $\mathfrak{X}$, the canonical map

$$
Q \operatorname{Comp}(\mathscr{L},-1)^{\text {nondeg }} \rightarrow Q \mathcal{P}(\mathscr{L},-1)^{\text {nondeg }}
$$

is a weak equivalence. In particular, there is a morphism

$$
Q \mathcal{P}(\mathscr{L},-1)^{\text {nondeg }} \rightarrow G \operatorname{Sp}(\mathfrak{X},-1)
$$

in the homotopy category of simplicial sets.

Proof. This is much the same as Proposition 1.38. Lemma 2.22 combines with the obstruction maps

$$
\left(Q \operatorname{Comp}(\mathscr{L},-1) / G^{k}\right) \times_{\left(Q \mathcal{P}(\mathscr{L},-1) / G^{k}\right)}^{h}\left(Q \mathcal{P}(\mathscr{L},-1) / G^{k+1}\right) \rightarrow \operatorname{Ob}(Q \operatorname{Comp} / Q \mathcal{P}, \mathfrak{X}, k)
$$

to give the weak equivalences

$$
\begin{aligned}
& \left(Q \operatorname{Comp}(\mathscr{L},-1)^{\text {nondeg }} / G^{k+1}\right) \\
& \quad \simeq\left(Q \operatorname{Comp}(\mathscr{L},-1) / G^{k}\right) \times_{\left(Q \mathcal{P}(\mathscr{L},-1) / G^{k}\right)}^{h}\left(Q \mathcal{P}(\mathscr{L},-1)^{\text {nondeg }} / G^{k+1}\right) .
\end{aligned}
$$




\section{J. P. PRIDHAM}

\section{Quantisation for derived Artin N-stacks}

In order to proceed further, we will make use of the étale resolutions of derived Artin stacks by stacky CDGAs given in [Pri17, §3]. We just extend the results of Section 1 from CDGAs to stacky CDGAs, and then the étale descent approach of Section 2 adapts immediately.

3.1. Stacky CDGAs. We now recall some definitions and lemmas from $[$ Pri17, $§ 3]$. From now on, we will regard the CDGAs encountered so far as chain complexes $\cdots \stackrel{\delta}{\rightarrow} A_{1} \stackrel{\delta}{\rightarrow} A_{0} \stackrel{\delta}{\rightarrow} \cdots$ rather than cochain complexes - this will enable us to distinguish easily between derived (chain) and stacky (cochain) structures.

Definition 3.1. A stacky CDGA is a chain cochain complex $A \bullet$ equipped with a commutative product $A \otimes A \rightarrow A$ and unit $k \rightarrow A$. Given a chain CDGA $R$, a stacky CDGA over $R$ is then a morphism $R \rightarrow A$ of stacky CDGAs. We write $D G d g \operatorname{CAlg}(R)$ for the category of stacky CDGAs over $R$ and $D G^{+} d g \operatorname{CAlg}(R)$ for the full subcategory consisting of objects $A$ concentrated in non-negative cochain degrees.

When working with chain cochain complexes $V_{\bullet}^{\bullet}$, we will usually denote the chain differential by $\delta: V_{j}^{i} \rightarrow V_{j-1}^{i}$ and the cochain differential by $\partial: V_{j}^{i} \rightarrow V_{j}^{i+1}$.

Definition 3.2. Say that a morphism $U \rightarrow V$ of chain cochain complexes is a levelwise quasiisomorphism if $U^{i} \rightarrow V^{i}$ is a quasi-isomorphism for all $i \in \mathbb{Z}$. Say that a morphism of stacky CDGAs is a levelwise quasi-isomorphism if the underlying morphism of chain cochain complexes is so.

LEMma 3.3 ([Pri17, Lemma 3.4]). There is a cofibrantly generated model structure on stacky $C D G A$ s over $R$ in which fibrations are surjections and weak equivalences are levelwise quasiisomorphisms.

There is a denormalisation functor $D$ from non-negatively graded CDGAs to cosimplicial algebras, with left adjoint $D^{*}$ as in [Pri10, Definition 4.20]. Given a cosimplicial chain CDGA $A$, the algebra $D^{*} A$ is then a stacky CDGA in non-negative cochain degrees. By [Pri17, Lemma 3.5], the functor $D^{*}$ is a left Quillen functor from the Reedy model structure on cosimplicial chain CDGAs to the model structure of Lemma 3.3.

Since $D A$ is a pro-nilpotent extension of $A^{0}$, when $\mathrm{H}_{<0}(A)=0$, we think of the hypersheaf $\mathbf{R S p e c} D A$ as a stacky derived thickening of the derived affine scheme $\mathbf{R S p e c} A^{0}$.

Definition 3.4. Given a chain cochain complex $V$, define the cochain complex $\hat{\operatorname{tot}} V \subset \operatorname{Tot}{ }^{\Pi} V$ by

$$
(\hat{\operatorname{Tot}} V)^{m}:=\left(\bigoplus_{i<0} V_{i-m}^{i}\right) \oplus\left(\prod_{i \geqslant 0} V_{i-m}^{i}\right)
$$

with differential $\partial \pm \delta$.

Definition 3.5. Given a stacky CDGA $A$ and $A$-modules $M$ and $N$ in chain cochain complexes, define internal Homs $\mathcal{H} \operatorname{Hom}_{A}(M, N)$ by

$$
\mathcal{H o m}_{A}(M, N)_{j}^{i}=\operatorname{Hom}_{A_{\#}^{\#}}\left(M_{\#}^{\#}, N_{\#[j]}^{\#[i]}\right)
$$

with differentials $\partial f:=\partial_{N} \circ f \pm f \circ \partial_{M}$ and $\delta f:=\delta_{N} \circ f \pm f \circ \delta_{M}$, where $V_{\#}^{\#}$ denotes the bigraded vector space underlying a chain cochain complex $V$. 


\section{QUANTISING (-1)-SHIFTED SYMPLECTIC STRUCTURES}

Then define the Hom complex $\underline{\operatorname{Hom}}_{A}(M, N)$ by

$$
\underline{\operatorname{Hom}}_{A}(M, N):=\operatorname{Tot} \mathcal{H o m} A(M, N) \text {. }
$$

Note that there is a multiplication $\underline{\operatorname{Hom}}_{A}(M, N) \otimes \underline{\operatorname{Hom}}_{A}(N, P) \rightarrow \underline{\operatorname{Hom}}_{A}(M, P)$.

Definition 3.6. A morphism $A \rightarrow B$ in $D G^{+} d g \mathrm{CAlg}(R)$ is said to be homotopy formally étale when the map

$$
\left\{\operatorname{Tot} \sigma^{\leqslant q}\left(\mathbf{L} \Omega_{A}^{1} \otimes_{A}^{\mathbf{L}} B^{0}\right)\right\}_{q} \rightarrow\left\{\operatorname{Tot} \sigma^{\leqslant q}\left(\mathbf{L} \Omega_{B}^{1} \otimes_{B}^{\mathbf{L}} B^{0}\right)\right\}_{q}
$$

on the systems of brutal cotruncations is a pro-quasi-isomorphism.

Combining [Pri17, Proposition 3.13] with [Pri13b, Theorem 4.15 and Corollary 6.35], every strongly quasi-compact derived Artin $N$-stack over $R$ can be resolved by a homotopy formally étale cosimplicial diagram in $D G^{+} d g \mathrm{CAlg}(R)$.

3.2. Quantised polyvectors. We now fix a stacky CDGA $A$ over a chain CDGA $R$.

Definition 3.7. Given $A$-modules $M$ and $N$ in chain cochain complexes, inductively define the filtered chain cochain complex $\mathcal{D} \operatorname{iff}(M, N)=\mathcal{D i f f}_{A / R}(M, N) \subset \mathcal{H} \mathrm{om}_{R}(M, N)$ of differential operators from $M$ to $N$ by setting

(1) $F_{0} \operatorname{Diff}(M, N)=\mathcal{H} \operatorname{om}_{A}(M, N)$;

(2) $F_{k+1} \operatorname{Diff}(M, N)=\left\{u \in \mathcal{H} \operatorname{om}_{R}(M, N):[a, u] \in F_{k} \mathcal{D} \operatorname{iff}(M, N) \forall a \in A\right\}$, where $[a, u]=$ $a u-(-1)^{\operatorname{deg} a \operatorname{deg} u} u a ;$

(3) $\operatorname{Diff}(M, N)=\underline{\lim }_{k} F_{k} \mathcal{D} \operatorname{iff}(M, N)$.

We then define the filtered cochain complex $\operatorname{Diff}(M, N)=\hat{\operatorname{Diff}}_{A / R}(M, N) \subset \underline{\operatorname{Hom}}_{R}(M, N)$ by $\hat{\operatorname{Diff}}(M, N):=\lim _{k} \hat{\operatorname{Tot}} F_{k} \mathcal{D} \operatorname{iff}(M, N)$.

Definition 3.8. Given an $A$-module $M$ in chain cochain complexes, write $\mathscr{D}(M)=\mathscr{D}_{A / R}(M):=$ $\hat{\operatorname{Diff}}_{A / R}(M, M)$, which we regard as a sub-DGAA of $\underline{\operatorname{Hom}}_{R}(M, M)$. We simply write $\mathscr{D}_{A}=\mathscr{D}_{A / R}$ for $\mathscr{D}_{A / R}(A, A)$.

Definition 3.9. Define a strict line bundle over $A$ to be an $A$-module $M$ in chain cochain complexes such that $M_{\#}^{\#}$ is a projective module of rank 1 over the bigraded-commutative algebra $A_{\#}^{\#}$ underlying $A$.

Definitions 1.10 and 1.11 then carry over verbatim to define quantised polyvectors over a stacky CDGA and the filtrations $\tilde{F}, G$, and $G * \tilde{F}$.

We now follow [Pri17, $\S 3.3$ ] in making the following assumptions on $A \in D G^{+} d g \operatorname{CAlg}(R)$ :

(1) For any cofibrant replacement $\tilde{A} \rightarrow A$ in the model structure of Lemma 3.3, the morphism $\Omega_{\tilde{A} / R}^{1} \rightarrow \Omega_{A / R}^{1}$ is a levelwise quasi-isomorphism.

(2) The $A^{\#}$-module $\left(\Omega_{A / R}^{1}\right){ }^{\#}$ in graded chain complexes is cofibrant (that is, it has the left lifting property with respect to all surjections of $A^{\#}$-modules in graded chain complexes).

(3) There exists an $N$ for which the chain complexes $\left(\Omega_{A / R}^{1} \otimes_{A} A^{0}\right)^{i}$ are acyclic for all $i>N$. These conditions are satisfied by $D^{*} O(X)$ for DG Artin hypergroupoids $X$. 


\section{J. P. PRIDHAM}

For stacky CDGAs of this form, Definition 1.18 adapts verbatim to define the $\operatorname{space} Q \mathcal{P}(M,-1)$ of $E_{0}$ quantisations of a strict line bundle $M$ and its twisted counterpart $Q^{\text {tw }} \mathcal{P}(M,-1)$. The second assumption gives us isomorphisms

$$
\begin{gathered}
F^{p-2 i} \widehat{\operatorname{Pol}}(A,-1) \hbar^{i} \rightarrow \operatorname{gr}_{G}^{i}(G * \tilde{F})^{p} Q \widehat{\operatorname{Pol}}(M,-1), \\
T_{\pi_{\Delta}} F^{p-2 i} \widehat{\operatorname{Pol}}(A,-1) \hbar^{i} \rightarrow \operatorname{gr}_{G}^{i}(G * \tilde{F})^{p} T_{\Delta} Q \widehat{\operatorname{Pol}}(M,-1) .
\end{gathered}
$$

The following is a slight generalisation of [Pri17, Definition 3.21].

Definition 3.10. Say that an $E_{0}$ quantisation $\Delta=\sum_{j \geqslant 2} \Delta_{j} \hbar^{j}$ of a strict line bundle $M$ over $A$ is non-degenerate if the map

$$
\Delta_{2}^{\sharp}: \operatorname{Tot}^{\Pi}\left(M^{0} \otimes_{A} \Omega_{A}^{1}\right) \rightarrow \underline{\operatorname{Hom}}_{A}\left(\Omega_{A}^{1}, M^{0}\right)[1]
$$

of is a quasi-isomorphism and $\operatorname{Tot}^{\Pi}\left(\Omega_{A}^{1} \otimes_{A} A^{0}\right)$ is a perfect complex over $A^{0}$.

Definitions 1.22 and 1.23 adapt verbatim, giving tangent spaces $T Q \mathcal{P}(M,-1), T Q \mathcal{P}(M$, -1) $/ G^{k}, T Q^{\mathrm{tw}} \mathcal{P}(M,-1), T Q^{\mathrm{tw}} \mathcal{P}(M,-1) / G^{k}$, and a canonical tangent vector

$$
\sigma=-\partial_{\hbar^{-1}}: Q \widehat{\operatorname{Pol}}(M,-1) \rightarrow T Q \widehat{\operatorname{Pol}}(M,-1) .
$$

\subsection{Generalised symplectic structures and compatible quantisations.}

Definition 3.11 ([Pri17, Definition 3.23]). Define the de Rham complex $\operatorname{DR}(A / R)$ to be the product total complex of the bicomplex

$$
\operatorname{Tot}^{\Pi} A \stackrel{d}{\rightarrow} \operatorname{Tot}^{\Pi} \Omega_{A / R}^{1} \stackrel{d}{\rightarrow} \operatorname{Tot}^{\Pi} \Omega_{A / R}^{2} \stackrel{d}{\rightarrow} \cdots,
$$

so the total differential is $d \pm \delta \pm \partial$.

We define the Hodge filtration $F$ on $\mathrm{DR}(A / R)$ by setting $F^{p} \mathrm{DR}(A / R) \subset \mathrm{DR}(A / R)$ to consist of terms $\operatorname{Tot}^{\Pi} \Omega_{A / R}^{i}$ with $i \geqslant p$.

We then similarly define $\operatorname{DR}^{\prime}(A / R)$ to be the (triple) product total complex

$$
\operatorname{DR}^{\prime}(A / R):=\operatorname{Tot}^{\Pi} N \hat{A}^{\bullet+1}
$$

regarded as a filtered DGAA over $R$, with $F^{p} \operatorname{DR}^{\prime}(A / R):=\operatorname{Tot}^{\Pi} N F^{p} \hat{A}^{\bullet+1}$. Definition 1.28 then carries over to give a filtration $\tilde{F}$ on $D R^{\prime}(A / R) \llbracket \hbar \rrbracket$.

Definition 1.30 carries over to give a space $G \operatorname{PreSp}(A / R,-1)$ of generalised $(-1)$-shifted presymplectic structures on $A / R$. We say that a generalised pre-symplectic structure $\omega$ is symplectic if its leading term $\omega_{0} \in \operatorname{PreSp}(A / R,-1)$ is symplectic in the sense of [Pri17, Definition 3.24]; explicitly, this says that $\operatorname{Tot}^{\Pi}\left(\Omega_{A}^{1} \otimes_{A} A^{0}\right)$ is a perfect complex over $A^{0}$ and the map

$$
\left[\omega_{0}\right]^{\sharp}: \underline{\operatorname{Hom}}_{A}\left(\Omega_{A}^{1}, A^{0}\right)[-n] \rightarrow \operatorname{Tot}^{\Pi}\left(\Omega_{A / R}^{1} \otimes_{A} A^{0}\right)
$$

is a quasi-isomorphism. We then let $\operatorname{Sp}(A / R, n) \subset \operatorname{PreSp}(A / R, n)$ consist of the symplectic structures; this is a union of path-components.

Lemmas 1.32 and 1.33 then adapt to give compatible maps

$$
\mu(-, \Delta): \mathrm{DR}^{\prime}(A / R) \llbracket \hbar \rrbracket / G^{k} \rightarrow T_{\Delta} Q \widehat{\operatorname{Pol}}(M,-1) / G^{k}
$$

respecting the filtrations $(G * \tilde{F})$.

We may now define the space $Q \operatorname{Comp}(A / R, n)$ of compatible quantisations as in Definition 1.36, with Proposition 1.38 adapting to show that

$$
Q \operatorname{Comp}(M,-1)^{\text {nondeg }} \rightarrow Q \mathcal{P}(M,-1)^{\text {nondeg }}
$$




\section{QUANTISING (-1)-SHIFTED SYMPLECTIC STRUCTURES}

is a weak equivalence and Proposition 1.41 adapting to show that the resulting maps

$$
\begin{aligned}
Q \mathcal{P}(M,-1)^{\text {nondeg }} / G^{k} & \rightarrow\left(Q \mathcal{P}(M,-1)^{\text {nondeg }} / G^{2}\right) \times{ }_{\left(G \operatorname{Sp}(A,-1) / G^{2}\right)}^{h}\left(G \operatorname{Sp}(A,-1) / G^{k}\right) \\
& \simeq\left(Q \mathcal{P}(M,-1)^{\text {nondeg }} / G^{2}\right) \times \prod_{i \geqslant 2} \underline{\operatorname{MC}}\left(\operatorname{DR}(A / R) \hbar^{i}\right)
\end{aligned}
$$

are weak equivalences for all $k \geqslant 2$.

3.4. Diagrams and derived Artin stacks. We now generalise the constructions of Section 2 to stacky CDGAs, allowing us to adapt arguments for DM stacks to apply to Artin stacks.

3.4.1. Diagrams. We may now proceed as in [Pri17, $\S 3.4 .2]$. For any small category $I$, any $I$-diagram $A$ in $D G^{+} d g \mathrm{CAlg}(R)$, and $A$-modules $M$ and $N$ in $I$-diagrams of chain cochain complexes, we define $\mathcal{D i f f}_{A / R}(M, N)$ to be the equaliser of

$$
\prod_{i \in I} \operatorname{Diff}_{A(i) / R}(M(i), N(i)) \Longrightarrow \prod_{f: i \rightarrow j \text { in } I} \operatorname{Diff}_{A(i) / R}\left(M(i), f_{*} N(j)\right)
$$

The constructions $Q \mathcal{P}(-,-1), G \operatorname{PreSp}(-,-1)$ and $Q \operatorname{Comp}(-,-1)$ all adapt to such diagrams and behave well for $[m]$-diagrams $A$ which are fibrant and cofibrant for the injective $[m]$-diagram model structure on stacky CDGAs, so $A(i)$ is cofibrant for the model structure of Lemma 3.3, and the maps $A(i) \rightarrow A(i+1)$ are all surjective. In particular, these constructions satisfy the conditions of [Pri17, §3.4.2], so for each construction $F$, we have an $\infty$ functor

$$
\mathbf{R} F: \mathbf{L} D G^{+} d g \mathrm{CAlg}(R)^{\text {ét }} \rightarrow \mathbf{L} s \operatorname{Set}
$$

on the $\infty$-categories given by localising weak equivalences, with $(\mathbf{R} F)(A) \simeq F(A)$ for all cofibrant stacky CDGAs $A$ over $R$. Here, $D G^{+} d g \mathrm{CAlg}(R)^{\text {ét }} \subset D G^{+} d g \mathrm{CAlg}(R)$ is the subcategory of morphisms $A \rightarrow B$ which are homotopy formally étale in the sense of Definition 3.6.

By the naturality of these constructions and the equivalences above, we then have weak equivalences of $\infty$-functors

$$
\mathbf{R} Q \operatorname{Comp}(-,-1)^{\text {nondeg }} \rightarrow \mathbf{R} Q \mathcal{P}(-,-1)^{\text {nondeg }}
$$

and

$$
\mathbf{R} Q \mathcal{P}(-,-1)^{\text {nondeg }} / G^{k} \rightarrow\left(\mathbf{R} Q \mathcal{P}(-,-1)^{\text {nondeg }} / G^{2}\right) \times_{\left(\mathbf{R} G \operatorname{Sp}(-,-1) / G^{2}\right)}^{h}\left(\mathbf{R} G \operatorname{Sp}(-,-1) / G^{k}\right)
$$

for all $k \geqslant 2$.

The approach of [Pri17, §3.4.2] now applies immediately to associate to any of the constructions above an $\infty$-functor on derived Artin $N$-stacks, with natural transformations and equivalences carrying over. However, this is not quite sufficient for our purposes since we wish to consider quantisations of non-trivial line bundles.

3.4.2. Descent and line bundles. We say that a morphism $A \rightarrow B$ in $D G^{+} d g \mathrm{CAlg}(R)$ is a covering if $A^{0} \rightarrow B^{0}$ is faithfully flat. In particular, this implies that

$$
\underset{i}{\operatorname{holim}} \mathbf{R S p e c} D^{i} B \rightarrow \underset{i}{\operatorname{holim}} \mathbf{R} \operatorname{Spec} D^{i} A
$$

is a surjection of étale hypersheaves. Note that when $X \rightarrow Y$ is a relative trivial derived Artin hypergroupoid, $X_{0} \rightarrow Y_{0}$ is faithfully flat, so the morphism $D^{*} O(Y) \rightarrow D^{*} O(X)$ is a covering in the sense above. 


\section{J. P. PRIDHAM}

In Section 2.3, we were able to extend the functor $Q \mathcal{P}$ to line bundles solely by making use of the $\mathbb{G}_{m}$-action on it. For Artin stacks, the situation is more subtle because for any $A \in$ $D G^{+} \operatorname{Alg}(\mathbb{Q})$, we have $\operatorname{Hom}\left(D^{*} O\left(B \mathbb{G}_{m}\right), A\right) \cong \mathrm{Z}^{1} A$.

The most naïve simplicial set-valued functor we can consider on $D G^{+} d g \operatorname{Alg}(\mathbb{Q})$ is $\left(B \mathbb{G}_{m}\right)^{\Delta}$ 。 $D$, which is represented by the cosimplicial CDGA $D^{*} O\left(\left(B \mathbb{G}_{m}\right)^{\Delta}\right)$ and sends $A$ to the nerve $B\left[\mathrm{Z}^{1}\left(\mathrm{Z}_{0} A\right) /\left(\mathrm{Z}_{0} A^{0}\right)^{\times}\right]$of the groupoid

$$
\operatorname{TLB}(A):=\left[\mathrm{Z}^{1}\left(\mathrm{Z}_{0} A\right) /\left(\mathrm{Z}_{0} A^{0}\right)^{\times}\right]
$$

where $f \in\left(A^{0}\right)^{\times}$acts on $\mathrm{Z}^{1} A$ by addition of $\partial \log f=f^{-1} \partial f$. We think of $\operatorname{TLB}(A)$ as the groupoid of trivial line bundles.

For any cofibrant $A \in D G^{+} d g \operatorname{Alg}(R)$, we can extend $Q \mathcal{P}$ to a simplicial representation of the groupoid $\operatorname{TLB}(A)$ above by sending an object $b \in \mathrm{Z}^{1}\left(\mathrm{Z}_{0} A\right)$ to $Q \mathcal{P}\left(A_{b},-1\right)$, with $\left(\mathrm{Z}_{0} A^{0}\right)^{\times}$ acting via functoriality for line bundles. Note that the quotient representation $Q \mathcal{P}(-,-1) / G^{1}=$ $\mathcal{P}(-,-1)$ is trivial; we also set $G$ PreSp to be a trivial representation $b \mapsto G \operatorname{PreSp}(A)$.

Definition 3.12. For any of the constructions $F$ of Section 3.4.1, let $\mathbf{R}\left(F /{ }^{h} \mathbb{G}_{m}\right)$ be the $\infty$ functor on $\mathbf{L} d g \mathrm{CAlg}(R)^{\text {ét }}$ given by applying the construction of [Pri17, $\left.\S 3.4 .2\right]$ to the right-derived functor of the Grothendieck construction

$$
A \mapsto \underset{b \in \operatorname{TLB}(A)}{\operatorname{holim}} F\left(A_{b}\right),
$$

then taking hypersheafification with respect to homotopy formally étale coverings.

Given a derived Artin $N$-stack $\mathfrak{X}$ and $A \in D G^{+} d g \operatorname{CAlg}(R)$, we say that an element $f \in$ $\operatorname{holim}_{i} \mathfrak{X}\left(D^{i} A\right)$ is homotopy formally étale if the induced morphism

$$
N_{c} f_{0}^{*} \mathbb{L}_{\mathfrak{X} / R} \rightarrow\left\{\operatorname{Tot} \sigma^{\leqslant q} \mathbf{L} \Omega_{A / R}^{1} \otimes_{A}^{\mathbf{L}} A^{0}\right\}_{q}
$$

from [Pri17, $\S 3.2 .2]$ is a pro-quasi-isomorphism. We then write $\left(d g_{+} D G\right.$ Aff $\left._{\text {ét }} \downarrow \mathfrak{X}\right)$ for the $\infty$ category of homotopy formally étale elements $f \in \operatorname{holim}_{i} \mathfrak{X}\left(D^{i} A\right)$ with homotopy formally étale maps $A \rightarrow B$ between them.

Definition 3.13. Given a derived Artin $N$-stack $\mathfrak{X}$, a line bundle $\mathscr{L}$ on $\mathfrak{X}$, and any of the functors $F$ above, define $F(\mathscr{L})$ to be the homotopy limit of

$$
\mathbf{R}\left(F /{ }^{h} \mathbb{G}_{m}\right)(A) \times{ }_{\mathbf{R}\left(* / h \mathbb{G}_{m}\right)(A)}^{h}\left\{\left.\mathscr{L}\right|_{A}\right\}
$$

over the $\infty$-category $\left(d g_{+} D G\right.$ Aff $\left._{\text {ét }} \downarrow \mathfrak{X}\right)$.

If we now fix a derived Artin $N$-stack $\mathfrak{X}$, Definition 2.21 carries over verbatim to give a notion of non-degeneracy for a $(-1)$-shifted Poisson structure $\pi \in \mathcal{P}(\mathfrak{X},-1)$, and Propositions 2.20 and 2.23 readily adapt (substituting the relevant results from [Pri17, §3]), giving the following result.

Proposition 3.14. For any line bundle $\mathscr{L}$ on $\mathfrak{X}$, the canonical maps

$$
\begin{aligned}
Q \operatorname{Comp}(\mathscr{L},-1)^{\text {nondeg }} & \rightarrow Q \mathcal{P}(\mathscr{L},-1)^{\text {nondeg }}, \\
Q \operatorname{Comp}(\mathscr{L},-1) & \rightarrow\left(Q \operatorname{Comp}(\mathscr{L},-1) / G^{2}\right) \times{ }_{\left(G \operatorname{PreSp}(\mathfrak{X},-1) / G^{2}\right)}^{h} G \operatorname{PreSp}(\mathfrak{X},-1) \\
& \simeq\left(Q \operatorname{Comp}(\mathscr{L},-1) / G^{2}\right) \times \prod_{i \geqslant 2} \underline{\operatorname{MC}}\left(\operatorname{DR}(\mathfrak{X} / R) \hbar^{i}\right) .
\end{aligned}
$$




\section{QUANTISING (-1)-SHIFTED SYMPLECTIC STRUCTURES}

are filtered weak equivalences. In particular, there is a morphism

$$
Q \mathcal{P}(\mathscr{L},-1)^{\text {nondeg }} \rightarrow G \mathrm{Sp}(\mathfrak{X},-1)
$$

in the homotopy category of simplicial sets.

\section{Self-dual quantisations}

We now introduce the notion of duality for quantisations and indicate how it leads to canonical quantisations for line bundles which are Grothendieck-Verdier self-dual, giving rise to the perverse sheaf $\mathcal{P} \mathcal{V}$ of vanishing cycles from $\left[\mathrm{BBD}^{+} 15\right]$. From our point of view, the key property of this sheaf is that it is Verdier self-dual $\left[\mathrm{BBD}^{+} 15\right.$, Equation (2.6)], while the object it quantises is Grothendieck-Verdier self-dual.

4.1. Duality. We wish to consider line bundles $\mathscr{L}$ equipped with an involutive equivalence $\mathscr{D}(\mathscr{L}) \simeq \mathscr{D}(\mathscr{L})^{\text {opp }}$.

Example 4.1. Following Remark 1.3, the bar construction for left $\mathscr{D}_{A}$-modules is just the completion of the Cech nerve along the diagonal. This gives an equivalence between left $\mathscr{D}_{X}$-modules and quasi-coherent sheaves on the stratified site of $X$ in the sense of [Gro68] (equivalently, on the presheaf $\left.X_{\text {strat }}(B):=\operatorname{Im}\left(X(B) \rightarrow X\left(\mathrm{H}_{0} B^{\text {red }}\right)\right)\right)$. Since $\underline{\operatorname{Hom}}_{A}(A \hat{\otimes} A, M)=\operatorname{pr}_{1}^{!} M$, where ! denotes exceptional pullback, it also means that right $\mathscr{D}$-modules correspond to !-quasi-coherent sheaves.

Since there is a natural map from the stratified site to the infinitesimal site, this means that left and right crystals in the sense of [GR14] give rise to left and right $\mathscr{D}$-modules in our sense, and in particular that the dualising complex $\omega_{X}$ on $X$ naturally has the structure of a right $\mathscr{D}$-module.

A derived scheme $X$ is said to be Gorenstein when $\omega_{X}$ is a line bundle, and if we write

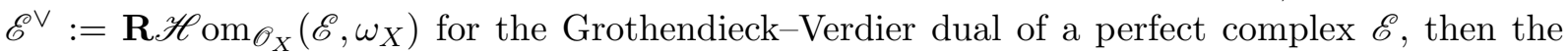
right $\mathscr{D}$-module structure of $\omega_{X}$ gives a quasi-isomorphism of DGAAs between $\mathscr{D}(\mathscr{E})^{\text {opp }}$ and $\mathscr{D}\left(\mathscr{E}^{\vee}\right)$. Thus an equivalence between $\mathscr{D}(\mathscr{L})$ and $\mathscr{D}(\mathscr{L})^{\text {opp }}$ is the same as $\mathscr{D}(\mathscr{L}) \simeq \mathscr{D}\left(\mathscr{L}^{\vee}\right)$, which is satisfied when $\mathscr{L} \simeq \mathscr{L}^{\vee}\left(\right.$ so $\mathscr{L}$ is a square root of $\left.\omega_{X}\right)$.

Indeed, an equivalence will exist whenever $\mathscr{L}$ has the structure of a right $\mathscr{D}(\mathscr{L})$-module or, equivalently, whenever $\mathscr{L}^{\otimes 2}$ has the structure of a right $\mathscr{D}$-module; the equivalence will automatically be involutive as $\mathscr{L}$ has rank 1 .

Definition 4.2. For a line bundle $\mathscr{L}$ with a right $\mathscr{D}$-module structure on $\mathscr{L}^{\otimes 2}$, we write $(-)^{t}: \mathscr{D}(\mathscr{L})^{\text {opp }} \rightarrow \mathscr{D}(\mathscr{L})$ for the natural quasi-isomorphism induced by the quasi-isomorphism $\mathscr{D}^{\mathrm{opp}} \simeq \mathscr{D}\left(\mathscr{L}^{\otimes 2}\right)$ given by the right $\mathscr{D}$-module structure. We then define

$$
(-)^{*}: Q \widehat{\operatorname{Pol}}(\mathscr{L},-1) \rightarrow Q \widehat{\operatorname{Pol}}(\mathscr{L},-1) \quad \text { by } \quad \Delta^{*}(\hbar):=-\Delta^{t}(-\hbar) .
$$

Since this is a quasi-isomorphism of filtered DGLAs, it gives rise to a weak equivalence

$$
(-)^{*}: Q \widehat{\operatorname{Pol}}(\mathscr{L},-1) \rightarrow Q \widehat{\operatorname{Pol}}(\mathscr{L},-1),
$$

and hence

$$
Q \mathcal{P}(\mathscr{L},-1) \rightarrow Q \mathcal{P}(\mathscr{L},-1)
$$

The reason for the choice of sign $-\hbar$ in the definition of $\Delta^{*}$ is that on the associated graded 


\section{J. P. PRIDHAM}

piece $\operatorname{gr}_{p}^{F} \mathscr{D}_{X}(\mathscr{E}) \cong \operatorname{Symm}^{p} \mathscr{T}_{X}$, the operation $(-)^{t}$ is given by $(-1)^{p}$. Thus the underlying Poisson structures satisfy $\pi_{\Delta^{*}}=\pi_{\Delta}$.

Definition 4.3. For a line bundle $\mathscr{L}$ with $\mathscr{L}^{\otimes 2}$ a right $\mathscr{D}$-module, the map $(-)^{*}$ becomes a (homotopy) involution of $Q \widehat{\operatorname{Pol}}(\mathscr{L},-1)$, and we define $Q \widehat{\mathrm{Pol}}(\mathscr{L},-1)^{\text {sd }}$ to be the space of homotopy fixed points of the resulting $\mathbb{Z} / 2$-action.

Similarly, we define the space

$$
Q \mathcal{P}(\mathscr{L},-1)^{\mathrm{sd}}
$$

of self-dual quantisations to be the space of homotopy fixed points of the $\mathbb{Z} / 2$-action on $Q \mathcal{P}(\mathscr{L},-1)$ generated by $(-)^{*}$.

Remark 4.4. Following Remark 1.13, to each $E_{0}$ quantisation $\Delta \in Q \mathcal{P}(\mathscr{L},-1)$, there corresponds a right $\mathscr{D}_{X} \llbracket \hbar \rrbracket$-module $\mathscr{M}_{\hbar}:=\left(\mathscr{L}_{\otimes_{\mathscr{O}_{X}} \mathbf{L}} \mathscr{D}_{X} \llbracket \hbar \rrbracket, \delta+\Delta \cdot\{-\}\right)$. Definition 4.3 says that the quantisation is self-dual with respect to the right $\mathscr{D}$-module structure on $\mathscr{L}^{\otimes 2}$ when

$$
\mathbf{R} \mathscr{H} \operatorname{om}_{\mathscr{D}_{X}^{\text {opp }} \llbracket \hbar \rrbracket}\left(\mathscr{M}_{\hbar}, \mathscr{D}_{X} \llbracket \hbar \rrbracket\right) \otimes \mathscr{O}_{X} \mathscr{L}^{\otimes 2}
$$

is equivalent to $\mathscr{M}_{-\hbar}$ as a right $\mathscr{D}$-module. This can be phrased as a symmetric perfect pairing

$$
\left(\mathscr{M}_{-\hbar} \otimes_{\mathscr{O}_{X}}^{\mathbf{L}} \mathscr{L}^{-1}\right) \otimes_{\mathscr{D}_{X}(\mathscr{L}) \llbracket \hbar \rrbracket}^{\mathbf{L}}\left(\mathscr{M}_{\hbar} \otimes_{\mathscr{O}_{X}}^{\mathbf{L}} \mathscr{L}^{-1}\right)^{\text {opp }} \rightarrow \mathscr{D}(\mathscr{L}) \llbracket \hbar \rrbracket .
$$

Lemma 4.5. For the filtration $G$ induced on $\tilde{F}^{p} Q \widehat{\mathrm{Pol}}(\mathscr{L},-1)^{\text {sd }}$ by the corresponding filtration on $\tilde{F}^{p} Q \widehat{\operatorname{Pol}}(\mathscr{L},-1)$, we have

$$
\operatorname{gr}_{G}^{k} \tilde{F}^{p} Q \widehat{\operatorname{Pol}}(\mathscr{L},-1)^{\mathrm{sd}} \simeq \begin{cases}\operatorname{gr}_{G}^{k} \tilde{F}^{p} Q \widehat{\operatorname{Pol}}(\mathscr{L},-1), & k \text { even }, \\ 0, & k \text { odd }\end{cases}
$$

Proof. As already observed, the involution acts trivially on $\operatorname{gr}_{G}^{0} Q \widehat{\operatorname{Pol}}(\mathscr{L},-1)$. It therefore acts as multiplication by $(-1)^{k}$ on $\operatorname{gr}_{G}^{k} Q \widehat{\operatorname{Pol}}(\mathscr{L},-1)=\hbar^{k} \operatorname{gr}_{G}^{0} Q \widehat{\operatorname{Pol}}(\mathscr{L},-1)$.

In particular, this means that $\operatorname{Ob}(Q \mathcal{P}, \mathscr{L}, 1)^{\mathrm{sd}} \simeq 0$, so the map

$$
Q \mathcal{P}(\mathscr{L},-1)^{\mathrm{sd}} / G^{2} \rightarrow Q \mathcal{P}(\mathscr{L},-1) / G^{1} \simeq \mathcal{P}(X,-1)
$$

is a weak equivalence. In other words, Poisson structures correspond to first-order self-dual quantisations. We can say much more in non-degenerate cases.

Proposition 4.6. For a line bundle $\mathscr{L}$ with $\mathscr{L}^{\otimes 2}$ a right $\mathscr{D}$-module (such as any square root of $\left.\omega_{X}\right)$, there is a canonical weak equivalence

$$
Q \mathcal{P}(\mathscr{L},-1)^{\text {nondeg }, s d} \simeq \mathcal{P}(X,-1)^{\text {nondeg }} \times \underline{\mathrm{MC}}\left(\hbar^{2} \mathrm{DR}(X / R) \llbracket \hbar^{2} \rrbracket\right) .
$$

In particular, every non-degenerate $(-1)$-shifted Poisson structure gives a canonical choice of self-dual quantisation of $\mathscr{L}$.

Proof. Lemma 4.5 implies that we have weak equivalences

$$
\begin{gathered}
Q \mathcal{P}(\mathscr{L},-1)^{\mathrm{sd}} / G^{2 i} \rightarrow Q \mathcal{P}(\mathscr{L},-1)^{\mathrm{sd}} / G^{2 i-1}, \\
Q \mathcal{P}(\mathscr{L},-1)^{\mathrm{sd}} / G^{2 i+1} \rightarrow\left(Q \mathcal{P}(\mathscr{L},-1)^{\mathrm{sd}} / G^{2 i}\right) \times_{\left(Q \mathcal{P}(\mathscr{L},-1) / G^{2 i}\right)}^{h}\left(Q \mathcal{P}(\mathscr{L},-1) / G^{2 i+1}\right) .
\end{gathered}
$$

Combined with Propositions 1.41 and 2.20, these give weak equivalences

$$
Q \mathcal{P}(\mathscr{L},-1)^{\text {nondeg }, s d} / G^{2 i+1} \rightarrow\left(Q \mathcal{P}(\mathscr{L},-1)^{\text {nondeg }, s d} / G^{2 i}\right) \times \underline{\mathrm{MC}}\left(\hbar^{2 i} \operatorname{DR}(X / R)\right)
$$




\section{QUANTISING (-1)-SHIFTED SYMPLECTIC STRUCTURES}

for all $i>0$, so

$$
\begin{aligned}
Q \mathcal{P}(\mathscr{L},-1)^{\text {nondeg }, s d} / G^{2 i+1} & \simeq\left(Q \mathcal{P}(\mathscr{L},-1)^{\text {nondeg }, s d} / G^{2 i}\right) \times \underline{\mathrm{MC}}\left(\hbar^{2 i} \operatorname{DR}(X / R)\right) \\
& \simeq Q \mathcal{P}(\mathscr{L},-1)^{\text {nondeg }} / G^{2 i-1} \times \underline{\mathrm{MC}}\left(\hbar^{2 i} \operatorname{DR}(X / R)\right),
\end{aligned}
$$

and we have seen that $*$ acts trivially on $Q \mathcal{P}(\mathscr{L},-1) / G^{1}=\mathcal{P}(\mathscr{L},-1)$, so $Q \mathcal{P}(\mathscr{L},-1)^{\text {sd }} / G^{1} \simeq$ $\mathcal{P}(\mathscr{L},-1)$.

Remark 4.7. The proof of Proposition 4.6 only shows that for a self-dual quantisation of a nondegenerate (-1)-shifted Poisson structure, the corresponding generalised symplectic structure is determined by its even coefficients. In fact, the odd coefficients must be homotopic to 0, with the following reasoning.

As $\mu$ is multiplicative and the de Rham algebra is commutative, we have a homotopy $\mu(\omega, \Delta)^{t} \simeq \mu\left(\omega,-\Delta^{t}\right)$ for any $\omega$ and $\Delta$, so $\mu(\omega, \Delta)^{t}(-\hbar) \simeq \mu\left(\omega, \Delta^{*}\right)(\hbar)$. We also have $\sigma(\Delta)^{t}(-\hbar)$ $\simeq \sigma\left(\Delta^{*}\right)(\hbar)$, so $\omega(\hbar)$ is compatible with $\Delta$ if and only if $\omega(-\hbar)$ is compatible with $\Delta^{*}$. When $\Delta$ is self-dual and non-degenerate, this implies that $\omega(\hbar) \simeq \omega(-\hbar)$.

For a more explicit description of the generalised symplectic structure $\omega$ corresponding to a non-degenerate self-dual quantisation $\Delta$, observe that we then have an isomorphism

$$
\begin{aligned}
\mu(-, \Delta): \mathrm{H}^{*} & \left(F^{2} \mathrm{DR}(X / R) \times \hbar^{2} \mathrm{DR}(X / R) \llbracket \hbar^{2} \rrbracket\right) \\
& \rightarrow\left\{v \in \mathrm{H}^{*}\left(T_{\Delta}(G * \tilde{F})^{2} Q \widehat{\operatorname{Pol}}(\mathscr{L},-1)\right): v(-\hbar)=v^{t}(\hbar)\right\}
\end{aligned}
$$

and that $[\omega]$ must be the inverse image of $\left[\hbar^{2} \partial \Delta / \partial \hbar\right]$.

4.2. Vanishing cycles. Given a smooth scheme $Y$ of dimension $m$ over $\mathbb{C}$ and a function $f: Y \rightarrow \mathbb{A}^{1}$, we can consider the derived critical locus $X$ of $f$, which is equipped with a canonical $(-1)$-shifted symplectic structure $\omega$. Explicitly, $\mathscr{O}_{X}$ is the CDGA given by the alternating algebra $\mathscr{O}_{Y}\left[\mathscr{T}_{Y}[1]\right]$, with differential $\delta$ given by contraction with $d f$.

Now, the line bundle $\Omega_{Y}^{m}$ pulls back to give a square root $i^{*} \Omega_{Y}^{m}$ of the dualising sheaf $\omega_{X}$ on $X$. This complex can be written explicitly as $i^{*} \Omega_{Y}^{m} \cong\left(\Omega_{Y}^{*}[m], d f \wedge\right)$. There is a canonical (-1)shifted symplectic structure $\omega$ on $X$, and we write $\Delta_{\omega}$ for the unique compatible self-dual $E_{0}$ quantisation of Proposition 4.6.

Lemma 4.8. On the derived critical locus $X$, the quantisation $\Delta_{\omega}$ is given by $\hbar d: \Omega_{Y}^{*} \rightarrow \Omega_{Y}^{*} \llbracket \hbar \rrbracket$ for the de Rham differential $d$ on $Y$.

Proof. We first need to check that $\Delta:=\Delta_{\omega}$ is self-dual, but this follows because we have a pairing

$$
\left(i^{*} \Omega_{Y}^{m} \llbracket \hbar \rrbracket, \delta+\hbar d\right) \otimes_{R}\left(i^{*} \Omega_{Y}^{m} \llbracket \hbar \rrbracket, \delta-\hbar d\right) \rightarrow\left(i^{*} \Omega_{Y}^{m}\right)^{\otimes 2} \llbracket \hbar \rrbracket=\omega_{X} \llbracket \hbar \rrbracket
$$

given by combining the cup product $\Omega_{Y}^{*}[m] \otimes \Omega_{Y}^{*}[m] \rightarrow \Omega_{Y}^{*}[2 m]$ with projection to $\Omega_{Y}^{m}[m]$ followed by inclusion in $\omega_{X} \cong \Omega_{Y}^{m} \otimes_{\mathscr{O}_{Y}} \Omega_{Y}^{*}[m]$.

Now, for $y \in \mathscr{O}_{Y} \subset \mathscr{O}_{X}$, the differential operator $[\Delta, y]$ acts on $\Omega_{Y}^{*}[m]$ as multiplication by $\hbar d y$. For $\eta \in \mathscr{T}_{Y} \subset \mathscr{O}_{X}$, the differential operator $[\Delta, \eta]$ acts on $\Omega_{Y}^{*}[m]$ as the Lie derivative $\hbar$ Lie $_{\eta}$. Thus the ring homomorphism $\mu(-, \Delta)$ is given on generators of $\Omega_{X}^{1}$ by

$$
\mu(d y, \Delta)=\hbar d y \wedge, \quad \mu(d \eta, \Delta)=\hbar \operatorname{Lie}_{\eta},
$$

and we need to show that it maps $\omega$ to $\hbar^{2} \partial \Delta / \partial \hbar=\hbar^{2} d$, up to homotopy.

There is a canonical representative $\omega \in \mathrm{Z}^{-1} \Gamma\left(X, \Omega_{X}^{2}\right)$ with $d \omega=0$, and on any affine open, we may lift it to an element of $\tilde{\omega} \in \mathrm{Z}^{1} F^{2} \mathrm{DR}^{\prime}(X / R)$ lying in $N^{2} \hat{\mathscr{O}}_{X}^{\otimes \bullet+1}$. Any such choices differ 


\section{J. P. PRIDHAM}

by $d \alpha$ for $\alpha \in F^{2} N^{1} \hat{\mathscr{O}}_{X}^{\otimes \bullet+1}$; in other words, $\alpha \in I^{2}$ for $I=\operatorname{ker}\left(\hat{\mathscr{O}}_{X}^{\otimes 2} \rightarrow \mathscr{O}_{X}\right)$. We now just observe that $\mu(\alpha, \Delta)$ is anti-self-dual since $\mu(d a . d b, \Delta)=\hbar\langle a, b\rangle_{\pi} \in \hbar \mathscr{O}_{X}$. Therefore, $\mu(\tilde{\omega}, \Delta)+\mu(\tilde{\omega}, \Delta)^{*}$ is strictly independent of the choice of lift.

It thus suffices to show that for some such local choice, we have $\mu(\tilde{\omega}, \Delta)=\sigma(\Delta)$. To make things explicit, we now take local coordinates $y_{1}, \ldots, y_{m}$ and write $\eta_{i} \in \mathscr{O}_{X}$ for the element given by $\partial_{y_{i}} \in \mathscr{T}_{Y}$, so $X$ has coordinates $y_{1}, \ldots, y_{m}, \eta_{1}, \ldots, \eta_{m}$. The generator $d y_{1} \wedge \cdots \wedge d y_{m}$ then gives an isomorphism $i^{*} \Omega_{Y}^{m} \cong \mathscr{O}_{X}$, and $\Delta$ corresponds to the quantisation of $\mathscr{O}_{X}$ given by $\Delta:=\hbar \sum_{i} \partial_{y_{i}} \partial_{\eta_{i}}$.

The calculations above reduce to

$$
\begin{aligned}
& \mu\left(y_{i} \otimes 1-1 \otimes y_{i}, \Delta\right)=\mu\left(d y_{i}, \Delta\right)=\hbar \partial_{\eta_{i}}, \\
& \mu\left(\eta_{i} \otimes 1-1 \otimes \eta_{i}, \Delta\right)=\mu\left(d \eta_{i}, \Delta\right)=\hbar \partial_{y_{i}} .
\end{aligned}
$$

We now choose the lift $\tilde{\omega}:=\sum_{i} d y_{i} \smile d \eta_{i} \in \mathrm{Z}^{1} F^{2} \mathrm{DR}^{\prime}(X / R)$ of the canonical (-1)-shifted symplectic structure $\omega=\sum_{i} d y_{i} \wedge d \eta_{i} \in \mathrm{Z}^{1} F^{2} \mathrm{DR}(X / R)$. Since $\mu(-, \Delta)$ is multiplicative, it follows that $\mu\left(d y_{i} \smile d \eta_{i}, \Delta\right)=\hbar^{2} \partial_{\eta_{i}} \partial_{y_{i}}$, so

$$
\mu(\tilde{\omega}, \Delta)=\mu\left(\sum_{i} d y_{i} \smile d \eta_{i}, \Delta\right)=\sum_{i} \hbar^{2} \partial_{\eta_{i}} \partial_{y_{i}}=\hbar^{2} \frac{\partial \Delta}{\partial \hbar}=\sigma(\Delta) .
$$

Proposition 4.9. On the derived critical locus $(X, \omega)$, the localisation

$$
\mathbf{R} \Gamma\left(X,\left(M \llbracket \hbar \rrbracket, \delta+\Delta_{\omega}\right) \otimes_{\mathbb{C}[\hbar]} \mathbb{C}\left[\hbar, \hbar^{-1}\right]\right.
$$

is quasi-isomorphic to $\mathbf{R} \Gamma\left(X, \mathcal{P} \mathcal{V}_{Y, f}^{\bullet}\right)((\hbar))$ for the perverse sheaf $\mathcal{P} \mathcal{V}$ of vanishing cycles from $\left[\mathrm{BBD}^{+} 15, \S 2.4\right]$.

Proof. By [Sab10, Theorem 1.1], we have $\mathbf{R} \Gamma\left(X, \mathcal{P} \mathcal{V}_{Y, f}^{\bullet}\right)((u)) \simeq\left(\Omega_{Y}^{*}((u)), d-u^{-1} d f\right)[m]$. Multiplying by $\hbar^{i}$ in degree $i$ and setting $\hbar=-u$, we get $\mathbf{R} \Gamma\left(X, \mathcal{P} \mathcal{V}_{Y, f}^{\bullet}\right)((\hbar)) \simeq\left(\Omega_{Y}^{*}((\hbar)), d f+\hbar d\right)[m]$; Lemma 4.8 completes the proof.

4.3. Quantisation for $\boldsymbol{n}$-shifted symplectic structures. We now discuss how to generalise these results to more general $n$-shifted structures, including the non-trivial cases $n=0,-2$.

4.3.1. Unshifted Poisson structures. To address the case $n=0$, replace the filtered DGAA $\mathscr{D}_{A}$ of differential operators with the filtered DGLA $\mathscr{D}_{A}^{\text {poly }}[1]$ of polydifferential operators, setting $Q \widehat{\operatorname{Pol}}(A, 0):=\prod_{p \geqslant 0} F_{p} \mathscr{D}_{A}^{\text {poly }} \hbar^{p-1}$. As in [VdB07], the HKR isomorphism leads to a quasiisomorphism between $\mathscr{D}_{A}^{\text {poly }}$ and the Hochschild complex of $A$ over $R$.

For a quantisation

$$
\Delta \in Q \mathcal{P}(A, 0):=\underline{\mathrm{MC}}(Q \widehat{\operatorname{Pol}}(A, 0)[1]),
$$

the centre $T_{\Delta} Q \widehat{\operatorname{Pol}}(A, 0):=(\hbar Q \widehat{\operatorname{Pol}}(A, 0), \delta+[\Delta,-])$ then has the canonical structure of an $E_{2}$-algebra. A choice of formality isomorphism for $E_{2}$ will therefore give a $P_{2}$-algebra structure on $T_{\Delta} Q \widehat{\operatorname{Pol}}(A, 0)$, and we may define a CDGA map

$$
\mu(-, \Delta): \operatorname{DR}(A) \rightarrow T_{\Delta} Q \widehat{\operatorname{Pol}}(A, 0)
$$

determined on generators by $\mu(a, \Delta)=a$ and $\mu(d f, \Delta)=[\Delta, f]$ for $a, f \in A$. This will be a quasi-isomorphism when $\Delta$ is non-degenerate. 


\section{QUANTISING (-1)-SHIFTED SYMPLECTIC STRUCTURES}

Such a construction yields analogues of all the results in Sections 1 and 2, with a cohomological shift. In particular, there is a map from quantisations to $\prod_{k \geqslant 0} \mathrm{H}^{2}\left(F^{2-2 k} \mathrm{DR}(A)\right) \hbar^{k}$, and the analogue of Propositions 1.41 and 2.20 says that a deformation quantisation of a non-degenerate Poisson structure exists whenever it can be quantised to first order. The analogues of self-dual $E_{0}$ quantisations are DQ algebroid quantisations $\mathscr{B}_{\hbar}$ equipped with involutions $\mathscr{B}_{-\hbar} \simeq \mathscr{B}_{\hbar}^{\text {opp }}$; for details, see [Pri18a].

4.3.2. Positively shifted Poisson structures. For $n \geqslant 1$, we can likewise define $Q \widehat{\operatorname{Pol}}(A, n)$ in terms of shifted differential operators or $E_{n+1}$-Hochschild complexes over Rees $(\operatorname{DR}(A))$. However, the formality of $E_{n+1}$ should yield equivalences $Q \widehat{\operatorname{Pol}}(A, n) \simeq \widehat{\operatorname{Pol}}(A, n) \llbracket \hbar \rrbracket$ and $Q \mathcal{P}(A / R, n)$ $\simeq Q \mathcal{P}(A \llbracket \hbar \rrbracket / R \llbracket \hbar \rrbracket, n)$, making the analogues of Propositions 1.38 and 1.41 less interesting.

(We might also wish to quantise higher analogues of line bundles: for derived stacks, these should be classes in $\mathrm{H}^{n+2}\left(\mathfrak{X}, \mathbb{G}_{m}\right)$. For unbounded CDGAs $A$, the analogue of the strict line bundle $A_{b}$ of Definition 1.9 is the curved $E_{n+1}$-algebra $(A, c)$ for $c \in \mathrm{Z}^{n+2}(A)$; because $A$ is an $E_{\infty}$-algebra, its Lie bracket is trivial, so we still have $\delta^{2}=[c,-]$.)

4.3.3. (-2)-shifted Poisson structures. For $n \geqslant-1$, there is a canonical $E_{n+2}$-algebra quantisation $\hbar Q \operatorname{Pol}(A, n)$ of $\hbar \operatorname{Pol}(A, n)$ given by $(n+1)$-shifted differential operators, which is equipped with an $E_{n+2}$-algebra morphism $A \rightarrow \hbar Q \operatorname{Pol}(A, n)$. In order to adapt the techniques of this paper to (-2)-shifted symplectic structures, [Pri18b] starts with the data of an $E_{0}$ quantisation

$$
\hbar Q \widehat{\operatorname{Pol}}(A,-2):=(\hbar \widehat{\operatorname{Pol}}(A,-2) \llbracket \hbar \rrbracket, \delta+\Delta)
$$

of the $P_{0}$-algebra $\hbar \widehat{\operatorname{Pol}}(A,-2)$, with $\Delta \in Q \mathcal{P}(\hbar \operatorname{Pol}(A,-2),-1)$ satisfying $\Delta(A)=0$ (but not necessarily $A$-linear) and lifting the canonical Poisson bracket on $\hbar \widehat{\operatorname{Pol}}(A,-2)$.

In particular, the condition $\Delta(A)=0$ implies $\Delta(1)=0$, making $\hbar Q \widehat{\operatorname{Pol}}(A,-2)$ a $B V_{\infty^{-}}$ algebra. It thus suffices to have a right $\mathscr{D}$-module structure on $A$ and to define $\hbar Q \widehat{\operatorname{Pol}}(A,-2)$ in terms of the right de Rham complex of $A$, as in [Sch98]. However, (-2)-shifted symplectic derived schemes are seldom Gorenstein, so a right $\mathscr{D}$-module structure on $A$ is not necessarily equivalent to a left $\mathscr{D}$-module structure on the dualising complex $\omega_{A}$.

Given a $(-2)$-shifted Poisson structure $\pi \in \mathcal{P}(A,-2)$, an $E_{-1}$ quantisation of $\pi$ is defined [Pri18b] to be a lift of $\hbar \pi$ to an element $\hbar S$ of the $B V_{\infty}$-algebra $\hbar \tilde{F}^{2} Q \widehat{\operatorname{Pol}}(A,-2)$ satisfying the $L_{\infty}$ Maurer-Cartan equation or, equivalently, the quantum master equation $(\delta+\Delta) e^{S}=0$. When there exists a morphism $\operatorname{DR}^{r}(A) \rightarrow \mathrm{DR}^{r}\left(\omega_{A}\right)[-v \operatorname{dim} A]$ of right de Rham cohomology complexes, this gives rise to an element of degree $v \operatorname{dim} A$ in Borel-Moore homology, permitting comparison with [BJ17].

Write $\Delta_{S}:=\operatorname{ad}_{\left(e^{-S}\right)} \Delta+\delta S$; the centre $T_{S} Q \widehat{\operatorname{Pol}}(A,-2)$ of $S$ is defined to be the complex $\left(\hbar \widehat{\mathrm{Pol}}(A,-2) \llbracket \hbar \rrbracket, \delta+\Delta_{S}\right)$. We can then define a compatibility map $\mu$ by composing the map

$$
\mathrm{DR}^{\prime}(A) \rightarrow \mathrm{DR}^{\prime}(\operatorname{Pol}(A,-2)) \stackrel{\mu\left(-, \Delta_{S}\right)}{\longrightarrow} T_{\Delta_{S}} Q \widehat{\operatorname{Pol}}(\operatorname{Pol}(A,-2),-1)
$$

from this paper with the evaluation of differential operators at 1 . Since $\Delta_{S}(1)=0$ by the quantum master equation, this gives a filtered map

$$
\mu(-, S): \operatorname{DR}^{\prime}(A) \rightarrow T_{S} Q \widehat{\operatorname{Pol}}(A,-2),
$$

and a (-2)-shifted pre-symplectic structure $\omega$ is then said to be compatible with $S$ when $\mu(\omega, S) \simeq$ 


\section{J. P. PRIDHAM}

$\hbar^{2} \partial S / \partial \hbar$.

To first order, we have $\Delta_{S} \cong \Delta+\{\pi,-\}$; since $\Delta(A)=0$, the map $\mu(-, S): \mathrm{DR}^{\prime}(A) \rightarrow$ $T_{S} Q \widehat{\operatorname{Pol}}(A,-2) / G^{1}=T_{\pi} \widehat{\operatorname{Pol}}(A,-2)$ is then just the compatibility map $\mu(-, \pi)$ from [Pri17].

4.3.4. Self-duality. In Section 4, the key to self-duality for $E_{0}$ quantisations of $\sqrt{\omega}_{X}$ is the filtered involution $(-)^{t}: \mathscr{D}_{X}\left(\sqrt{\omega}_{X}\right) \simeq \mathscr{D}_{X}\left(\sqrt{\omega}_{X}\right)^{\text {opp }}$. For $n \geqslant 0$, HKR isomorphisms mean that the analogue of $\mathscr{D}_{A}$ is the higher Hochschild cohomology complex $\mathrm{HH}^{E_{n+1}}(A)$ with its $E_{n+2^{-}}$ algebra structure. In order to define self-dual quantisations, we would thus need a filtered involution $(-)^{t}: \mathrm{HH}^{E_{n+1}}(A) \simeq \mathrm{HH}^{E_{n+1}}(A)^{\text {opp }}$, lifting the $P_{n+2}$-algebra involution of $\widehat{\operatorname{Pol}}(X, n)$ given by $(-1)^{m}$ on $m$-vectors.

Of course, when $n>0$, the equivalence $Q \widehat{\operatorname{Pol}}(A, n) \simeq \widehat{\operatorname{Pol}}(A, n) \llbracket \hbar \rrbracket$ coming from the formality of $E_{n+1}$ allows us to transfer the involution on $\widehat{\operatorname{Pol}}(A, n)$ to $Q \widehat{\operatorname{Pol}}(A, n)$. We then have $Q \widehat{\operatorname{Pol}}(A, n)^{\text {sd }} \simeq \widehat{\operatorname{Pol}}(A, n) \llbracket \hbar^{2} \rrbracket$, giving a sense in which the canonical quantisations coming from formality of $E_{n+1}$ are self-dual.

Whereas Grothendieck-Verdier self-duality for a line bundle $\mathscr{L}$ is an involutive equivalence $\mathscr{L} \simeq \mathbf{R} \mathcal{H} \mathrm{m}_{\mathscr{O}_{X}}\left(-, \omega_{X}\right)$, the obvious notion of self-duality for an algebroid $\mathcal{A}$ is an involution $\mathcal{A} \simeq \mathcal{A}^{\text {opp }}$. When $n=0$, an involutive filtered $E_{2}$-equivalence on the Hochschild complex of $X$ gives an analogue of Proposition 4.6, generating self-dual quantisations from symplectic structures. This amounts to looking for DQ algebroid quantisations $\mathcal{A}$ equipped with involutions $\mathcal{A}(-\hbar) \simeq \mathcal{A}(\hbar)^{\text {opp }}$ deforming a chosen contravariant involution on the Picard algebroid (or even any 2-line bundle). Such involutions correspond to line bundles, the obvious choices being $\mathbf{R} \mathcal{H}_{\mathrm{om}_{\mathscr{O}_{X}}}\left(-, \mathscr{O}_{X}\right)$ and $\mathbf{R} \mathcal{H o m}_{\mathscr{O}_{X}}\left(-, \omega_{X}\right)$, and involutive 2-line bundles are $\mu_{2}$-gerbes. For more details, see [Pri18a].

\section{REFERENCES}

BBD $^{+} 15$ C. Brav, V. Bussi, D. Dupont, D. Joyce, and B. Szendröi, Symmetries and stabilization for sheaves of vanishing cycles (with an appendix by Jörg Schürmann), J. Singul. 11 (2015), 85151; doi:10.5427/jsing.2015.11e.

Beh09 K. Behrend, Donaldson-Thomas type invariants via microlocal geometry, Ann. of Math. 170 (2009), no. 3, 1307-1338; doi:10.4007/annals.2009.170.1307.

Beh14_, On the virtual fundamental class, Proceedings of the International Congress of Mathematicians - Seoul 2014, Vol. II, (Kyung Moon Sa, Seoul, 2014), 591-613; http://www . icm2014. org/download/Proceedings_Volume_II.pdf.

BJ17 D. Borisov and D. Joyce, Virtual fundamental classes for moduli spaces of sheaves on CalabiYau four-folds, Geom. Topol. 21 (2017), no. 6, 3231-3311; doi:10.2140/gt.2017.21.3231.

GR14 D. Gaitsgory and N. Rozenblyum, Crystals and D-modules, Pure Appl. Math. Q. 10 (2014), no. 1,57-154; doi:10.4310/PAMQ.2014.v10.n1.a2.

Gro68 A. Grothendieck, Crystals and the de Rham cohomology of schemes, in Dix exposés sur la cohomologie des schémas, Adv. Stud. Pure Math., vol. 3 (North-Holland, Amsterdam, 1968), 306-358.

Hin01 V. Hinich, DG coalgebras as formal stacks, J. Pure Appl. Algebra 162 (2001), no. 2-3, 209-250; doi:10.1016/S0022-4049(00)00121-3.

Kra00 O. Kravchenko, Deformations of Batalin-Vilkovisky algebras, Poisson Geometry (Warsaw, 1998), Banach Center Publ., vol. 51 (Polish Acad. Sci. Inst. Math., Warsaw, 2000), 131-139. 


\section{QuANTISING (-1)-SHIFTED SYMPLECTIC STRUCTURES}

Pos11 L. Positselski, Two kinds of derived categories, Koszul duality, and comodulecontramodule correspondence, Mem. Amer. Math. Soc. 212 (2011), no. 996; doi: 10.1090/S0065-9266-2010-00631-8.

Pri10 J.P. Pridham, Unifying derived deformation theories, Adv. Math. 224 (2010), no. 3, 772-826; doi:10.1016/j.aim.2009.12.009. Corrigendum, Adv. Math. 228 (2011), no. 4, 2554-2556; doi:10.1016/j.aim.2011.07.004.

Pri13a Constructing derived moduli stacks, Geom. Topol. 17 (2013), no. 3, 1417-1495; doi: 10.2140/gt.2013.17.1417.

Pri13b_ Presenting higher stacks as simplicial schemes, Adv. Math. 238 (2013), 184-245; doi: 10.1016/j.aim.2013.01.009.

Pri17 _ Shifted Poisson and symplectic structures on derived N-stacks, J. Topol. 10 (2017), no. 1, 178-210; doi:10.1112/topo.12004.

Pri18a_, Deformation quantisation for unshifted symplectic structures on derived Artin stacks, Selecta Math. (N.S.) 24 (2018), no. 4, 3027-3059; doi:10.1007/s00029-018-0414-2.

Pri18b Deformation quantisation for (-2)-shifted symplectic structures, 2018, arXiv:1809. 11028.

PTVV13 T. Pantev, B. Toën, M. Vaquié, and G. Vezzosi, Shifted symplectic structures, Publ. Math. Inst. Hautes Études Sci. 117 (2013), no. 1, 271-328; doi:10.1007/s10240-013-0054-1.

Sab10 C. Sabbah, On a twisted de Rham complex, II, 2010, arXiv:1012.3818.

Sch98 V. Schechtman, Remarks on formal deformations and Batalin-Vilkovisky algebras, 1998, arXiv: math. AG/9802006.

Toë14 B. Toën, Derived algebraic geometry and deformation quantization, Proceedings of the International Congress of Mathematicians - Seoul 2014, Vol. II (Kyung Moon Sa, Seoul, 2014), 769-792; http://www.icm2014.org/download/Proceedings_Volume_II.pdf.

TV08 B. Toën and G. Vezzosi, Homotopical algebraic geometry. II. Geometric stacks and applications, Mem. Amer. Math. Soc. 193 (2008), no. 902; doi:10.1090/memo/0902.

VdB07 M. Van den Bergh, On global deformation quantization in the algebraic case, J. Algebra 315 (2007), no. 1, 326-395; doi:10.1016/j.jalgebra.2007.02.012.

J.P. Pridham j.pridham@ed.ac.uk

School of Mathematics and Maxwell Institute of Mathematics, University of Edinburgh, James Clerk Maxwell Building, The King's Buildings, Mayfield Road, Edinburgh, EH9 3FD, UK 